\title{
Imprint of magnetic flux expulsion at the core-mantle boundary on geomagnetic field intensity variations
}

Troyano, M.; Fournier, A.; Gallet, Y.; Finlay, C. C.

Published in:

Geophysical Journal International

Link to article, DOI:

10.1093/gji/ggaa126

Publication date:

2020

Document Version

Publisher's PDF, also known as Version of record

Link back to DTU Orbit

Citation (APA):

Troyano, M., Fournier, A., Gallet, Y., \& Finlay, C. C. (2020). Imprint of magnetic flux expulsion at the core-mantle boundary on geomagnetic field intensity variations. Geophysical Journal International, 221(3), 1984-2009. https://doi.org/10.1093/gij/ggaa126

\section{General rights}

Copyright and moral rights for the publications made accessible in the public portal are retained by the authors and/or other copyright owners and it is a condition of accessing publications that users recognise and abide by the legal requirements associated with these rights.

- Users may download and print one copy of any publication from the public portal for the purpose of private study or research.

- You may not further distribute the material or use it for any profit-making activity or commercial gain

- You may freely distribute the URL identifying the publication in the public portal 


\title{
Imprint of magnetic flux expulsion at the core-mantle boundary on geomagnetic field intensity variations
}

\author{
M. Troyano ${ }^{\oplus},{ }^{1}$ A. Fournier ${ }^{\oplus},{ }^{1}$ Y. Gallet ${ }^{\oplus 1}$ and C.C. Finlay ${ }^{\oplus 2}$ \\ ${ }^{1}$ Université de Paris, Institut de Physique du Globe de Paris, CNRS, F-75005 Paris, France.E-mail: troyano@ipgp.fr \\ ${ }^{2}$ DTU Space, Technical University of Denmark, 2800 Kongens Lyngby, Copenhagen, Denmark
}

Accepted 2020 March 13. Received 2020 March 13; in original form 2019 December 6

\begin{abstract}
S UMMAR Y
During the last decade, rapid or extreme geomagnetic field intensity variations associated with rates greater than the maximum currently observed have been inferred from archeomagnetic data in the Near-East and in Western Europe. The most extreme events, termed geomagnetic spikes, are defined as intensity peaks occurring over a short time (a few decades), and are characterized by high variation rates, up to several $\mu \mathrm{T} \mathrm{yr}^{-1}$. Magnetic flux expulsion from the Earth's outer core has been suggested as one possible explanation for these peaks but has not yet been examined in detail. In this study, we develop a 2-D kinematic model for magnetic flux expulsion whose key control parameter is the magnetic Reynolds number $R_{m}$, the ratio of magnetic diffusion time to advection time. This model enables the tracking of magnetic field lines which are distorted and folded by a fixed flow pattern. Two processes govern the magnetic evolution of the system. The first is the expulsion of magnetic flux from closed streamlines, whereby flux gradually concentrates near the boundaries of the domain, which leads to an increase of the magnetic energy of the system. If the upper boundary separates the conducting fluid from an insulating medium, a second process then takes place, that of diffusion through this interface, which we can quantify by monitoring the evolution of the vertical component of magnetic induction along this boundary. It is the conjunction of these two processes that defines our model of magnetic flux expulsion through the core-mantle boundary. We analyse several configurations with varying flow patterns and magnetic boundary conditions. We first focus on flux expulsion from a single eddy. Since this specific configuration has been widely studied, we use it to benchmark our implementation against analytic solutions and previously published numerical results. We next turn our attention to a configuration which involves two counter-rotating eddies producing an upwelling at the centre of the domain, and comprises an upper boundary with an insulating medium. We find that the characteristic rise time and maximum instantaneous variation rate of the vertical component of the magnetic field that escapes the domain scale like $\sim R_{m}^{0.15}$ and $\sim R_{m}^{0.45}$, respectively. Extrapolation of these scaling laws to the Earth's régime is compared with various purported archeointensity highs reported in the Near-East and in Western Europe. According to our numerical experiments magnetic flux expulsion is unlikely to produce geomagnetic spikes, while intensity peaks of longer duration (one century and more) and smaller variation rates appear to be compatible with this process.
\end{abstract}

Key words: Dynamo: theories and simulations; Magnetic field variations through time; Rapid time variations; Archaeomagnetism.

\section{INTRODUCTION}

Studying the variations of the Earth's magnetic field provides invaluable information on the dynamics in the Earth's outer core through the magnetohydrodynamic equations. These variations combine two processes: advection and diffusion. Considering that the typical timescale for diffusion is much longer than that for advection, diffusion is often neglected, especially when dealing with short-term geomagnetic variations. Such a simplification is referred to as the frozen-flux approximation (Roberts \& Scott 1965). However, the validity of this approach is sometimes questioned (see Jackson \& Finlay 2015, for a review). First, the diffusion term is negligible 
compared to the advection term only when considering the same large length scale for both processes $(\sim 1000 \mathrm{~km})$, which does not hold for peculiar flow and/or field configurations, such as that occurring when an upwelling drives expulsion of toroidal magnetic field. From the observational standpoint, constraints on the processes underlying secular variation come from the study of maps of the radial component of the magnetic field at the core-mantle boundary $(\mathrm{CMB})$ inferred from data at the Earth's surface using inverse modelling (Jackson \& Finlay 2015). These models have sometimes been interpreted as suggesting local failures of the frozen-flux approximation, for example related to the growth or decay in intensity of magnetic flux patches at the $\mathrm{CMB}$, or the emergence of newly formed reverse flux patches (see Finlay et al. 2010, for a review). The outputs of numerical dynamo simulations also point to a significant contribution of radial diffusion to the secular variation at a regional scale (Amit \& Christensen 2008). This regional field-diffusion in specific locations is often interpreted as being due to flux expulsion events : the toroidal magnetic field confined within the outer core is advected by an upwelling of the flow, and concentrated at the CMB through which it then diffuses (Christensen \& Olson 2003; Christensen \& Wicht 2015). Flux expulsion thereby contributes to the emergence of reverse flux patches or the weakening/strenghtening of existing flux patches.

Flux expulsion is often invoked to explain episodes of notable field intensity change in specific places such as the growth of the South Atlantic anomaly and associated dipole decay since 1850 (Gubbins 1987; Gubbins et al. 2006), the acceleration of the drift of the north magnetic pole since the 1990s (Chulliat et al. 2010), or extreme geomagnetic field intensity variation rates as detected in archeomagnetic data sets (Shaar et al. 2011). In the latter case, flux expulsion has been tentatively proposed as an explanation for two episodes of extreme field intensity variations during the first half of the first millennium BC in the Near-East (see for example BenYosef et al. 2009; Shaar et al. 2011, 2016). These features, named geomagnetic spikes, involve extreme variation rates up to several $\mu \mathrm{T} \mathrm{yr}^{-1}$ and occur over only a few decades, a very short-time interval for such large changes. Livermore et al. (2014) emphasize that such large rates are difficult to reconcile with our current understanding of core dynamics. An optimized core surface flow, given the estimated available energy, could generate a pointwise rate-ofchange of geomagnetic intensity on the order of or lower than 1 $\mu \mathrm{T} \mathrm{yr}^{-1}$, while the maximum observed in present-day variations is $\sim 0.1 \mu \mathrm{T} \mathrm{yr}^{-1}$ (Olsen et al. 2014). Davies \& Constable (2018) study outputs of numerical dynamo simulations and find spike-like features, whose intensity variation rates reach $\sim 0.75 \mu \mathrm{T} \mathrm{yr}^{-1}$, corresponding to the lower-end of the values recently reassessed by Ben-Yosef et al. (2017), i.e. between 0.75 and $1.5 \mu \mathrm{T} \mathrm{yr}^{-1}$. Their study suggests that these events are linked to the intensification and migration of intense magnetic flux patches at the CMB. They also underline that current numerical dynamo simulations are not able to establish the occurrence of faster intensity variations at Earth-like parameters. Korte \& Constable (2018) draw a similar conclusion from the analysis of series of global field models. The origin of such extreme intensity variations thus remains elusive; in particular, the imprint at Earth's surface of magnetic flux expulsion in the core remains to be studied in detail.

The process of flux expulsion from closed streamlines (or more generally, of the expulsion of a scalar from closed streamlines) has been widely studied. The simplest case to analyse is the kinematic one, which involves only the induction equation. The canonical configuration is that of a prescribed circular flow which interacts with an initially horizontal magnetic field in a closed domain surrounded with a perfect conductor. Weiss (1966) and Charbonneau (2013) performed the theoretical and numerical analysis of this setup, in a 2-D Cartesian geometry. In the situation when advection dominates diffusion, the initially horizontal magnetic field lines are stretched and folded by the flow; this leads to an increase of the magnetic energy. Through this interaction, field lines are progressively expelled from the eddy by diffusion, and they concentrate near the edges of the domain, which leads ultimately to a global decrease of the total magnetic energy. This standard textbook configuration is characterized by a series of scaling laws relating for instance the timing and amplitude of the magnetic energy growth to the relative importance of transport by the flow to diffusion (quantified by the magnetic Reynolds number to be introduced below). In the case of the Earth, the setup departs from the textbook, as the mantle is to first order an insulator, which allows poloidal magnetic field lines to go through the CMB. Using a 2-D Cartesian model of a conducting fluid sandwiched between a lower perfectly conducting medium (the inner core in a first approximation) and an upper insulating medium (the mantle), and an imposed pair of counter-rotating eddies mimicking an upwelling, Bloxham (1986) demonstrated the plausibility of this process in the Earth's core, without attempting to derive scaling laws accounting for the characteristic timescale and magnitude of magnetic flux expulsion. Perhaps surprisingly, flux expulsion has not been systematically studied further in the decades since.

In this study, we investigate whether flux expulsion events at the $\mathrm{CMB}$ could be a viable mechanism to explain rapid or extreme field intensity variations as revealed by archeomagnetic data recently obtained in the Near-East and in Western Europe. We aim to derive scaling laws that provide detailed constraints on episodes of flux expulsion, with a particular focus on their duration. We will conduct this investigation in two dimensions, varying the geometry and the prescribed flows. First, we will follow Weiss (1966) and Charbonneau (2013) and consider only one eddy, the purpose being mostly to validate our numerical approach against previously published results. Then, a model similar to that of Bloxham (1986) involving two eddies is analysed. The results are compared with the maximum intensity variation rates as derived from different archeointensity data sets of different periods obtained in the Near-East and in Western Europe.

This paper is organized as follows: the numerical method is introduced in Section 2. The results are presented in Section 3, with a detailed analysis of the theoretical and numerical scaling laws for the expulsion of magnetic flux from closed streamlines. A comparison with results from previous studies is conducted in order to validate our numerical method. Then, magnetic flux expulsion through an insulating boundary is studied for different configurations in order to underline the effects of different flow geometry. Scaling laws are derived for these different configurations. In Section 4, the derived scalings laws are tentatively applied to the Earth and comparisons are made with various extreme archeointensity events inferred in the Near-East and in Western Europe. A conclusion follows in Section 5. An appendix provides the interested reader with the details of the numerical implementation and its verification against analytical solutions.

\section{MODEL AND METHOD}

We are interested in the kinematic interaction of fluid flow and magnetic field in a closed domain $\mathcal{D}$, surrounded by an exterior domain $\breve{\mathcal{D}}$. Two physical processes are at work. The first one is strictly speaking the flux expulsion process, that is the expulsion of 
magnetic flux from closed streamlines. This expulsion occurs when advection dominates over diffusion, namely when the frozen-flux approximation holds. This is a standard phenomenon in magnetohydrodynamics whose description can be found in classical textbooks (see e.g. Roberts 1967, Section 2.4), and whose numerical investigation was pioneered by Weiss (1966), who assumed that the outer region $\breve{\mathcal{D}}$ was filled by a perfect conductor. Rhines \& Young (1983) subsequently proposed an analytical solution to the expulsion of a passive scalar from closed streamlines which is suitable for a variety of fluid-flow configurations.

When magnetic flux gets concentrated towards the edges of the domain, it can radially diffuse through the boundary, if the medium across the boundary is either finitely conducting or insulating. Magnetic field lines separate from fluid flow and the field diffuses in $\breve{\mathcal{D}}$. This second process is of geophysical interest, as it can lead to a local increase (or decrease) of magnetic flux at the boundary. In the geophysical literature, flux expulsion often refers to the joint effect of flux concentration towards the boundary (in this case the CMB) and diffusion through it. It is the combination of these two processes that we seek to investigate.

Let $(x, y, z)$ denote Cartesian coordinates. As illustrated in Fig. 1, we consider a 2-D Cartesian domain $\mathcal{D}$ of width $L_{x}$ and height $L_{z}$, setting the top of the domain at $z=0$. We focus on three configurations (see Fig. 1). Configuration 1 (Fig. 1a) is akin to one of the setups chosen by Weiss (1966); it consists of a square $\mathcal{D}\left(L_{x}=L_{z}\right)$, filled by one vortex, between two perfect conductors. The system is periodic in the $x$-direction. The initial magnetic field is uniform and parallel to the $x$-axis. In this setup, only the flux expulsion from closed streamlines occurs, there is no subsequent diffusion through the boundary. We choose this setup, and a slightly modified version with a different streamfunction, previously studied by Charbonneau (2013) in order to confirm previously published results and to validate our numerical implementation. The streamfunctions are shown in Figs 1(a) and (b), respectively for Weiss (1966) and Charbonneau (2013), see the next section for details. For Configuration 2 (Fig. 1b), we introduce a single modification: the top boundary is assumed to be insulating. Consequently, diffusion through that boundary occurs after the initial expulsion phase, and we can isolate this effect in order to see if it has a global effect on the system, compared with Configuration 1 (for both streamfunctions). Finally, Configuration 3 (Fig. 1c) retains the same boundaries (insulating top and perfectly conducting bottom), in a different geometry and with a different flow pattern. The domain is now rectangular $\left(L_{x}=2 L_{z}\right.$ throughout) and it contains two counter-rotating eddies, which induce an upwelling in the centre of the domain. The initial magnetic field is again uniform and parallel to the $x$-axis. From the geophysical standpoint, this is the analog of the toroidal field in the vicinity of the CMB. This last configuration is the one designed and previously studied by Bloxham (1986).

Regardless of the configuration retained, the evolution of the system is governed by the induction equation

$\frac{\partial \mathbf{B}}{\partial t}=\nabla \times[\mathbf{u} \times \mathbf{B}-\eta \times \nabla \times \mathbf{B}]$,

where $\mathbf{B}$ is the magnetic flux induction (hereafter the magnetic field), $\mathbf{u}$ the fluid velocity and $\eta$ the magnetic diffusivity. In our 2-D framework, this equation can be conveniently recast in terms of the magnetic vector potential $\mathbf{A}$, such that $\mathbf{B}(x, z, t)=\nabla \times \mathbf{A}(x, z, t)$ and $\mathbf{A}=A(x, z, t) \hat{\boldsymbol{y}}$. If we further impose the Coulomb gauge $\nabla \cdot \mathbf{A}=0$, we obtain a scalar equation for $A$,

$\frac{\partial A}{\partial t}=-\mathbf{u} \cdot \nabla A+\eta \nabla^{2} A$, which has to be supplemented by initial and boundary conditions. The initial vector potential is $A(x, z, t=0)=-B_{0} z$, whereby $B_{0}$ defines the amplitude of the initial field, which is parallel to the $x$ axis (recall the blue arrows in Fig. 1). Boundary conditions depend on the configuration chosen (see above). The flow $\mathbf{u}$ is steady and expressed in terms of a streamfunction $\Psi(x, z)$, such that $\mathbf{u}=\nabla \times$ $\Psi(x, z) \hat{\boldsymbol{y}}$. We choose the advection time $L_{z} / U$ as the characteristic timescale, where $U$ is the characteristic fluid velocity. Variables are non-dimensionalized according to

$t^{*}=t \frac{U}{L_{z}}, \quad z^{*}=\frac{z}{L_{z}}, \quad A^{*}=\frac{1}{B_{0} L_{z}} A$,

in which starred variables are dimensionless. This allows us to rewrite eq. (2) in the following non-dimensional form

$\left[\frac{\partial}{\partial t^{*}}-\frac{1}{R_{m}}\left(\frac{\partial^{2}}{\partial x^{* 2}}+\frac{\partial^{2}}{\partial z^{* 2}}\right)\right] A^{*}=\frac{\partial \Psi^{*}}{\partial z^{*}} \frac{\partial A^{*}}{\partial x^{*}}-\frac{\partial \Psi^{*}}{\partial x^{*}} \frac{\partial A^{*}}{\partial z^{*}}$,

with

$R_{m}=\frac{U L_{z}}{\eta}$

the magnetic Reynolds number. The magnetic Reynolds number is the ratio of the characteristic timescales of diffusion and advection

$\tau_{\mathrm{d}}=\frac{L_{z}^{2}}{\eta}$

and

$\tau_{\mathrm{adv}}=\frac{L_{z}}{U}$,

respectively; $R_{m}$ is the sole control parameter for the kinematic problem of interest here. In what follows, the stars are omitted and, unless otherwise noted, time is expressed in units of advection time.

In order to approximate the solution of eq. (3) numerically, a pseudospectral method is applied using a Fourier expansion along the $x$-direction together with a second-order accurate finite difference scheme in the $z$-direction. The resulting semi-discrete problem is advanced in time by means of an implicit-explicit scheme, along the lines described by Bloxham (1986). A Fourier expansion in $x$ is chosen because, for those configurations which possess an insulating upper boundary, the connection with an exterior potential solution is conveniently expressed using such a global basis (again, see Bloxham 1986). The reader is referred to the Appendix for a complete description of the implementation of the method and a thorough analysis of its convergence properties.

\section{RESULTS}

For each configuration described above, we systematically vary $R_{m}$, and integrate eq. (2) until a steady state is reached. Snapshots of $A$ will document the expulsion of flux by the flow towards the edges of the domain $\mathcal{D}$; in addition, we will monitor the evolution of the $z$ component of the magnetic field at the upper boundary, $B_{z 0}(x, t)=$ $B_{z}(x, z=0, t)$, if that boundary is insulating (Configurations 2 and 3 ). In order to derive scaling laws describing flux expulsion, we shall follow the time evolution of the total magnetic energy in the domain, $\mathcal{E}_{B}$,

$\mathcal{E}_{B}(t)=\iint_{\mathcal{D}} \frac{\mathbf{B}^{2}(t)}{2} \mathrm{~d} \mathcal{D}$,

and for the insulating upper boundary configurations also the evolution of the maximum of $B_{z 0}(x, t)=B(x, z=0, t)$,

$B_{z 0}^{\max }(t)=\max _{x}\left|B_{z 0}(x, t)\right|$. 


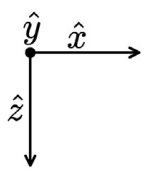

(a)

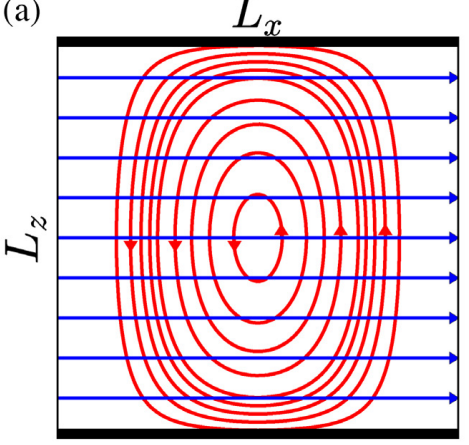

Configuration 1

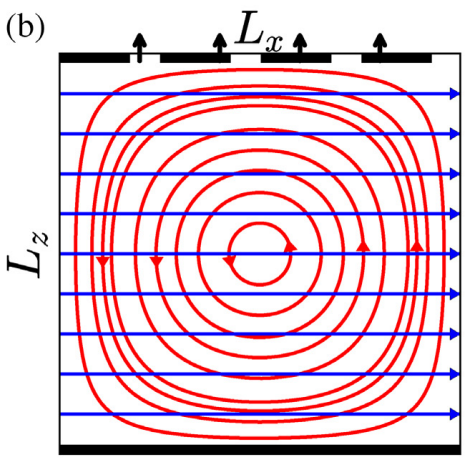

Configuration 2

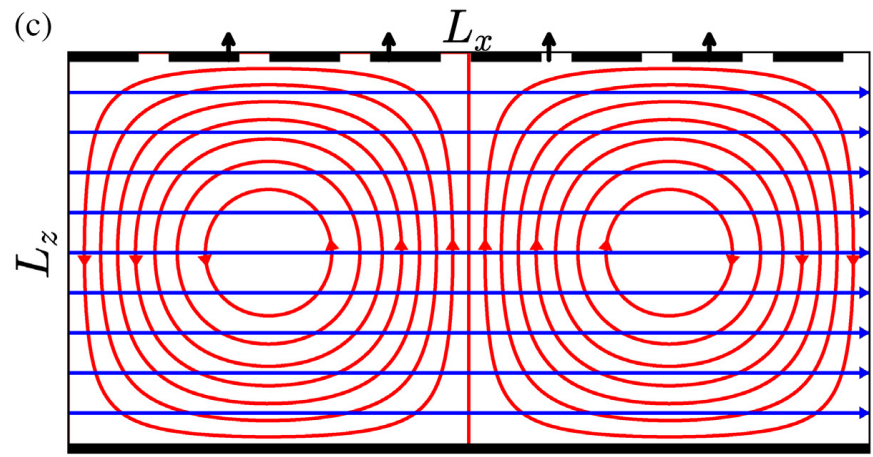

Configuration 3

Figure 1. Sketch of the three configurations considered in this study. (a) Configuration 1: square cell with perfectly conducting top and bottom boundaries, containing one anticlockwise vortex. (b) Configuration 2: square cell with an insulating top boundary and a perfectly conducting bottom boundary, containing one anticlockwise vortex. Note that the represented streamfunctions are different (see text for details). (c) Configuration 3: rectangular domain with insulating top boundary and perfectly conducting bottom boundary, containing two counter-rotating vortices. Blue and red arrows represent magnetic field lines and field flow, respectively. Solid and dashed black lines denote perfectly conducting and insulating boundaries, respectively.

To characterize the evolution of these two quantities, we track their maximum amplitude,

$\mathcal{E}_{B}^{\max }=\max _{t} \mathcal{E}_{B}(t), \quad$ and $\quad\left(B_{z 0}^{\max }\right)^{\max }=\max _{t} B_{z 0}^{\max }(t)$,

and the time $\tau_{\max }$ to reach the latter. The three different configurations each involves at least one perfectly conducting boundary (the lower one). As the magnetic field in a perfectly conducting medium is static, the magnetic field line on this boundary is kept fixed (see the Appendix for details). This condition prevents the magnetic energy from decaying, thereby enabling the establishment of a non-trivial steady state. We also characterize the steady state, defined as the moment at which the first order derivative in time of the monitored quantities remains under a threshold value close to zero. The criterion is set as follows:

$\left|\frac{\partial \mathcal{E}_{B}}{\partial t}\right|<C_{1}$, and $\quad\left|\frac{\partial\left(B_{z 0}^{\max }\right)^{\max }}{\partial t}\right|<C_{2}$ for $t \geq \tau_{\mathrm{ss}}$,

with $\tau_{\mathrm{ss}}$ the time at which the steady state is reached, and $C_{1}$ and $C_{2}$ two constants. Once $\tau_{\mathrm{ss}}$ is identified, the corresponding amplitude for each of the quantities is determined. The threshold value $C_{1}$ is fixed at $C_{1}=5 \times 10^{-3} \cdot C_{2}$ is fixed depending on the order of magnitude reached by $\left(B_{z 0}^{\max }\right)^{\max }$. We also determine the instantaneous rate of change of $B_{z 0}^{\max }$ at an instant $t_{n}$ defined as

$R\left(t_{n}\right)=\frac{\mathrm{d} B_{z 0}^{\max }}{\mathrm{d} t} \approx \frac{B_{z 0}^{\max }\left(t_{n+1}\right)-B_{z 0}^{\max }\left(t_{n}\right)}{\Delta t}$,

with $t_{n+1}-t_{n}=\Delta t=0.1 \tau_{\text {adv }}$; we track its maximum

$R^{\max }=\max _{t} R(t)$.
In the following section, we describe the results for the 3 chosen configurations. $\mathcal{E}_{B}^{\max }, \mathcal{E}_{B}^{\text {ss }}$ and $\tau_{\mathrm{ss}}$ are mainly used to compare our findings with those obtained in previous studies in order to validate our numerical implementation. Those previous studies do not investigate the behaviour of $\tau_{\max }$. Here, the quantities $\left(B_{z 0}^{\max }\right)^{\max }, \tau_{\max }$ and $R^{\max }$ are used to analyze the process of flux expulsion through an insulating boundary and to discuss this process with regards to extreme archeointensity events.

\subsection{Configuration 1: flux expulsion from one eddy with a perfectly conducting upper boundary}

This configuration presents a square domain $\mathcal{D}$ of unit length $L$ and contains a single counter-clockwise eddy. The top and bottom boundaries are perfectly conducting. The initial magnetic field is horizontal. The magnetic field lines are 'attached' to the left and right boundaries such that $A[x=(0, L), z, t]=A[x=$ $(0, L), z, t=0]$. Two different streamfunctions $\Psi$ are considered, following Weiss (1966, Fig. 2a), see Fig. 1(a) and Charbonneau (2013, chap. 2, Fig. 2.8), see Fig. 1(b)

$\Psi_{1}(x, z)=\frac{1}{\pi}\left(1-4 x^{2}\right)^{4} \sin (\pi z)$,
$\Psi_{2}(x, z)=\frac{1}{4 \pi}[1-\cos (2 \pi x)][1-\cos (2 \pi z)]$,

respectively. Figs 2(a)-(c) illustrate the evolution of the scalar potential $A$ after $0.5,2$ and 11 advection times respectively, for the streamfunction $\Psi_{1}$ and for a magnetic Reynolds number $R_{m}=1000$. On 
(a)

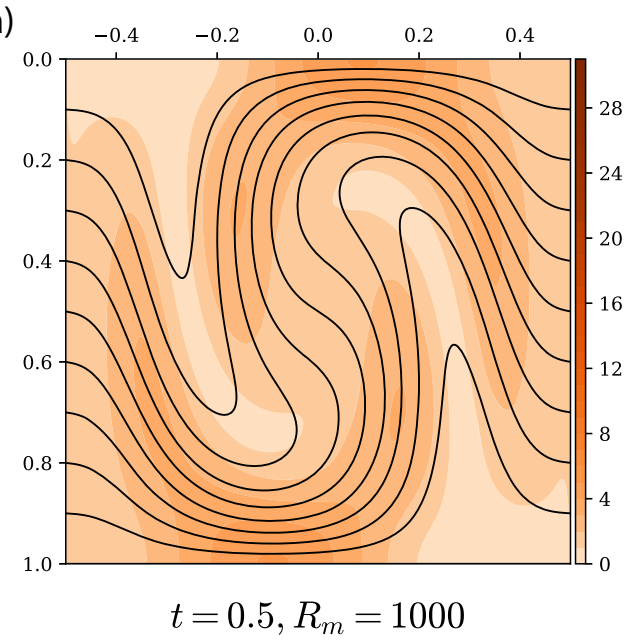

(c)

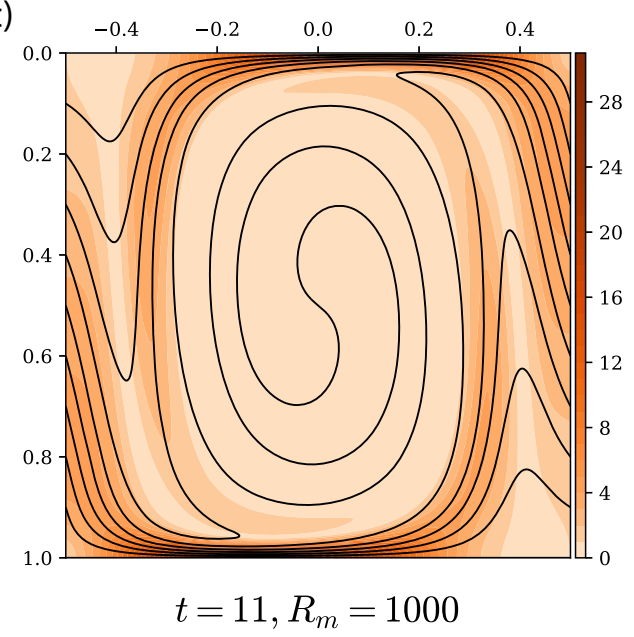

(b)

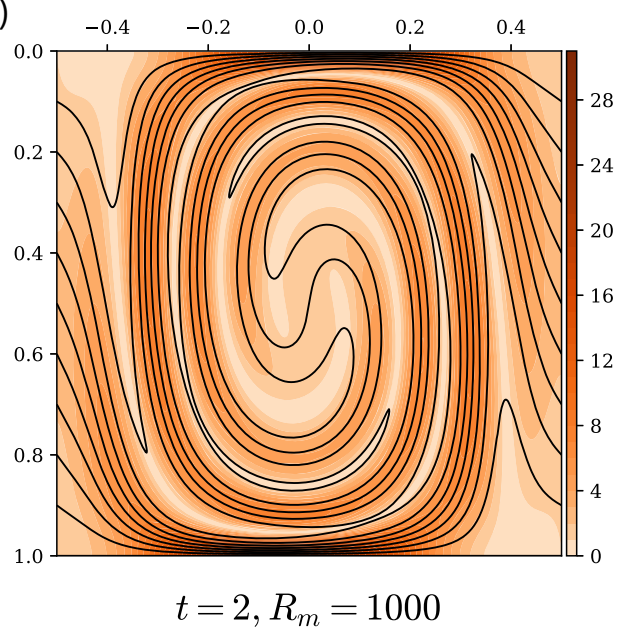

(d)

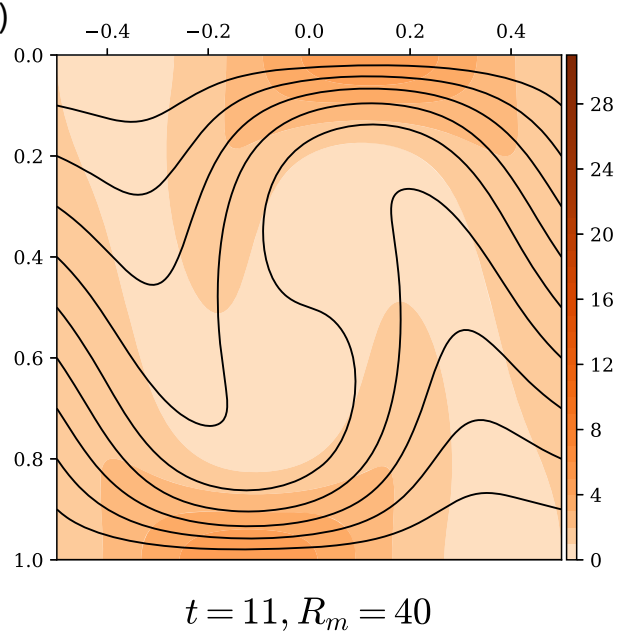

(e)

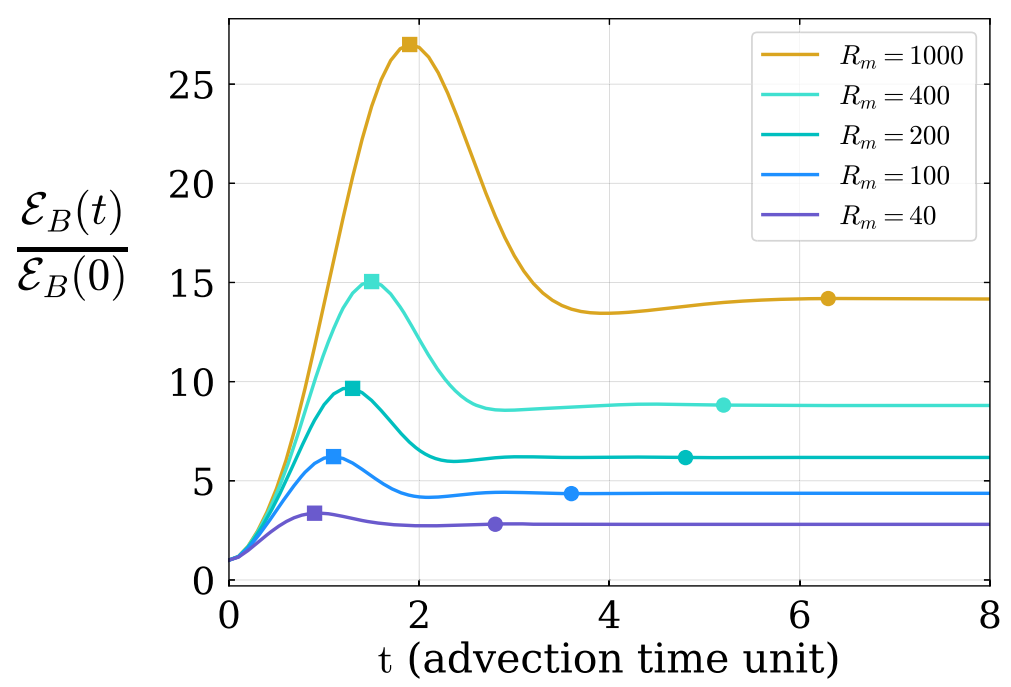

Figure 2. Time evolution of the magnetic field for Configuration 1 if the prescribed streamfunction is $\Psi_{1}$ (eq. 11). The black lines are iso-contours of the vector potential $A$ (therefore magnetic field lines), shown every $0.1 B_{0} L_{z}$. The colourscale represents the current magnetic field strength normalized by its initial value. The second row represents the magnetic field in the final stage (steady state) for a magnetic Reynolds number $R_{m}=1000$ (c) and for $R_{m}=40$ (d). (e) shows the temporal evolution of the magnetic energy normalized by its initial value for each computed $R_{m}$. The squares (resp. circles) denote the time when the maximum (resp. steady-state) stage of the evolution is reached. See text for details. 
each panel, isolines of $A$ correspond to magnetic field lines, and the colour scale represents the local strength of the magnetic field, $F=\sqrt{B_{x}^{2}+B_{z}^{2}}$. Likewise, Fig. 3 displays the same quantities when the chosen streamfunction is $\Psi_{2}$, all other parameters being the same. Figs 2(a) and (b) show that the initially horizontal field lines are wound by the eddy. This leads to the formation of magnetic stripes of alternating polarity whose width decreases with time, driving enhanced magnetic diffusion. After eleven advection times, the flux is expelled from the eye of the eddy and concentrated near the edges (Fig. 2c). This evolution is similar to the evolution obtained by Weiss (1966) for the same $R_{m}$. The initial shear of the field lines leads to an increase of $\mathcal{E}_{B}$, as shown in Fig. 2(e) [to be compared with Weiss (1966, Fig. 5)]. This first stage is dominated by the advection of the magnetic field. When magnetic diffusion becomes significant, the energy reaches a maximum $\mathcal{E}_{B}^{\max }$ and starts to decrease until a steady state is obtained. The comparison of the energy evolution determined in our study and in Weiss (1966) study (for the same range of $R_{m}$ ) shows the same behaviour with a slight difference in energy amplitude (see below).

We follow the analysis by Weiss (1966) to derive theoretical scaling laws describing the behaviour of $\mathcal{E}_{B}$. During the first phase of evolution, the velocity field builds the component of the magnetic field parallel to flow motions. This corresponds to an advective term of magnitude $U B_{0} / L$ in the induction eq. (1). In the meantime, as the magnetic field lines are wrapped, the stripes so formed become closer and closer to one another, leading to the decrease of the actual characteristic lengthscale of magnetic field variations, $\ell$, and thus inducing the gradual expulsion of the magnetic field towards the edges of the eddy (Fig. 2b) by reconnection of field lines. This expulsion is the product of magnetic diffusion in the fluid which becomes significant before the characteristic diffusion time is reached due to the decreasing lengthscale. The energy then reaches a maximum $\mathcal{E}_{B}^{\max }$ (see the squares in Fig. 2) found by equating the advective term previously discussed with the diffusive term in the induction eq. (1) based on the characteristic lengthscale of magnetic variations

$U B_{0} / L \sim \eta B / \ell^{2}$.

Replacing $\ell$ using the conservation of magnetic flux $B \ell \sim B_{0} L$, with $B$ the current magnetic field strength and $B_{0}$ its initial value, it follows that, at $t=\tau_{\max }$ (squares in Fig. 2), $B \sim R_{m}^{1 / 3} B_{0}$ and hence $\mathcal{E}_{B}^{\max } \sim R_{m}^{2 / 3} B_{0}^{2}$. As diffusion gradually balances advection, the energy starts to decrease, and at time $t=\tau_{\text {ss }}$ (see the circles in Fig. 2), a steady state is reached when all the flux has been expelled from the cell and the magnetic energy becomes constant. This final state is represented in Fig. 2(c). At that point, all the remaining flux is concentrated at the edges of the cell, inside a boundary layer of width $\ell_{\text {ss }}$. The local diffusion time

$\tau_{\mathrm{ld}} \sim \ell_{\mathrm{ss}}^{2} / \eta$

decreases until it reaches $\tau_{\mathrm{ld}} \sim \tau_{\mathrm{adv}}$, leading to $\ell_{\mathrm{ss}} \sim R_{m}^{-1 / 2} L$. All the magnetic energy is concentrated inside these boundary layers (Fig. 2c). Conservation of flux implies that in these boundary layers $B_{\mathrm{bl}} \sim R_{m}^{1 / 2} B_{0}$. The total magnetic energy in the domain finally scales as

$\mathcal{E}_{B}^{\mathrm{sS}} \sim R_{m}^{1 / 2} B_{0}^{2}$.

Rhines \& Young (1983) show that in general, the expulsion of a passive scalar presents two stages: a rapid stage of expulsion during which the scalar quantity is rapidly mixed along streamlines by advection and a slow stage during which this quantity is homogenized by diffusion. In the case we are interested in, the mixing along the streamlines leads directly to the homogenization of the field due to the initial condition (uniform magnetic field) and the geometry of the flow. As underlined by Moffatt \& Kamkar (1983), the effect of diffusion is cumulative which prevents the determination of a simple scaling by the evaluation of this term at time $t$ but requires an integration of the diffusion term from 0 to $t$. Their analysis leads to $\tau_{\mathrm{ss}} \sim R_{m}^{1 / 3} \tau_{\mathrm{adv}}$. It also prevents one from finding a scaling law for $\tau_{\max }$, that is the time at which diffusion starts to equilibrate advection but is still not predominant. However, this quantity should present a low dependency to $R_{m}$ as $\mathcal{E}_{B}^{\max }$ is reached quickly, after a few turnover times, even for large $R_{m}$ (Fig. 2e).

As stressed by Rhines \& Young (1983), the actual expulsion of the flux from the inside of the eddy towards its edges is a mechanism of 'shear-augmented dispersion'. During the stage of increasing magnetic energy, the field lines are stretched and wrapped by the flow. It leads to the formation of stripes of alternating polarity. If the magnetic Reynolds number is large $(\gtrsim 50)$, the advection time is small compared to the diffusion time and magnetic field lines are further wrapped before the equilibrium between advection and diffusion occurs. When two field lines of opposite polarity are separated by a distance smaller than the diffusion scale, diffusion leads to the destructive folding of the field (Charbonneau 2013). On the contrary, when the magnetic Reynolds number is low, advection is not strong enough to sufficiently fold magnetic field lines. In this case, the balance between advection and diffusion is reached before the destruction of the field (Fig. 2d) and the decrease of energy is consequently less marked (see Fig. 2e, obtained for $R_{m}=40$ ).

Fig. 4(a) illustrates the scaling laws (summarized in Table 1) that can be extracted from our suite of simulations. For comparison with the work of Weiss (1966), $R_{m}$ lies between 40 and $10^{3}$ (including larger values of $R_{m}$ does not alter our findings significantly).

The maximum value of the energy $\mathcal{E}_{B}^{\max }$ is found to scale as $R_{m}^{0.645 \pm 0.004}$; Weiss (1966) numerically finds $R_{m}^{0.59}$ over the same range of $R_{m}$. Our results are close to the theoretical scaling law, $R_{m}^{2 / 3}$. In addition, the value of the energy of the steady state $\mathcal{E}_{B}^{\text {ss }}$ scales as $R_{m}^{0.504 \pm 0.005}$. Weiss (1966) gives $R_{m}^{0.42}$, while the theoretical expectation is $R_{m}^{1 / 2}$, again close to our numerically determined value.

With regard to the time taken to reach this steady value, we find that it scales as $R_{m}^{0.254 \pm 0.026}$, while the theoretical scaling law is $R_{m}^{1 / 3}$, which is markedly different. Interestingly, if we use $\Psi_{2}$, the same characteristic time is found to scale as $R_{m}^{0.412 \pm 0.001}$ (see Fig. 4b). This quantity appears to be highly dependent on the prescribed streamfunction (this will be discussed in the following). Here the theoretical exponent lies in between the two numerical values. To summarize, our results are close to the results obtained by Weiss (1966). As Weiss (1966) does not provide the details of his computational method, the small discrepancies noted between his results and ours most probably arise from a difference in the numerical approach. Note that to further ensure the accuracy of our model, the results of additional tests are provided in the Appendix. We conclude this part by stating that the agreement between the theoretical expectations, previously published results and our numerical findings convinces us of the validity of our numerical implementation.

The streamfunction $\Psi_{2}$ gives qualitatively the same results as previously described (Figs 3a-d). The evolution of the field and of the magnetic energy are similar and correspond to the results presented by Charbonneau (2013, chap. 2, Fig. 2.9). However, the scaling laws that can be numerically extracted are somewhat different from the ones obtained with $\Psi_{1}$. Fig. 3(e) shows the evolution of the magnetic energy as a function of time and it is interesting to note that the amplitude of the resulting energy for the same $R_{m}$ is lower for $\Psi_{2}$ (see Fig. 3e, where the dashed lines show for comparison the 
(a)

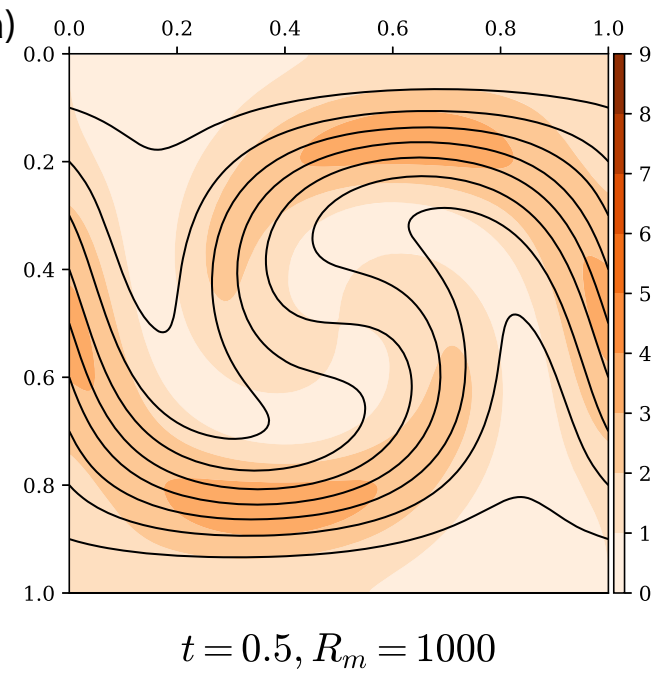

(c)

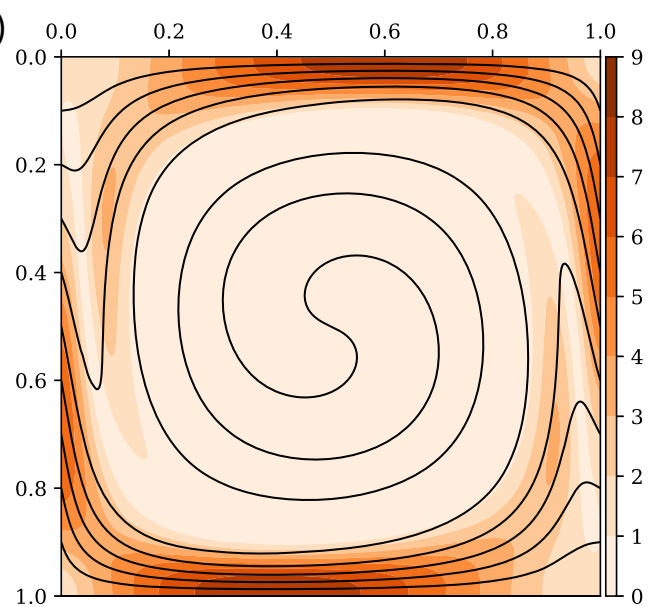

$t=11, R_{m}=1000$ (b)

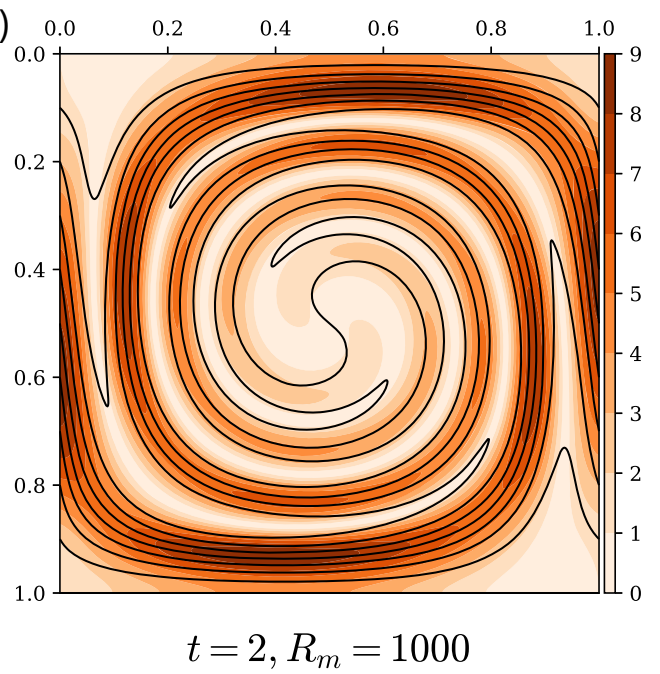

(d)

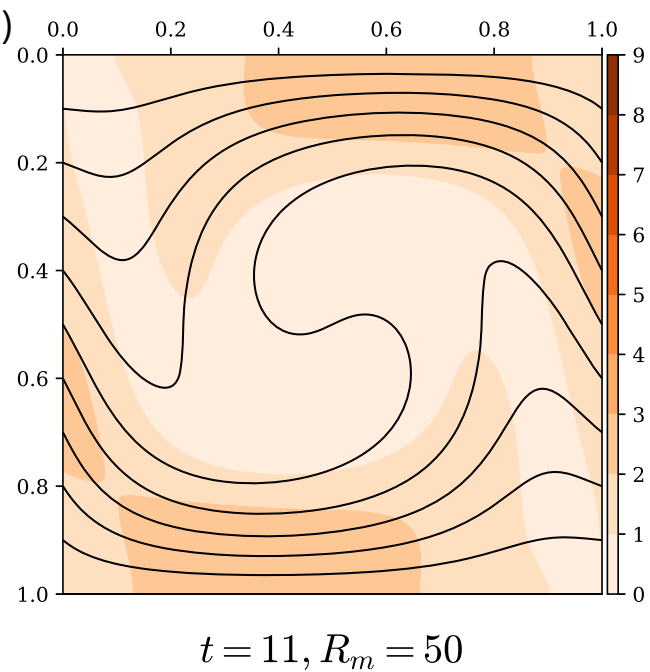

(e)

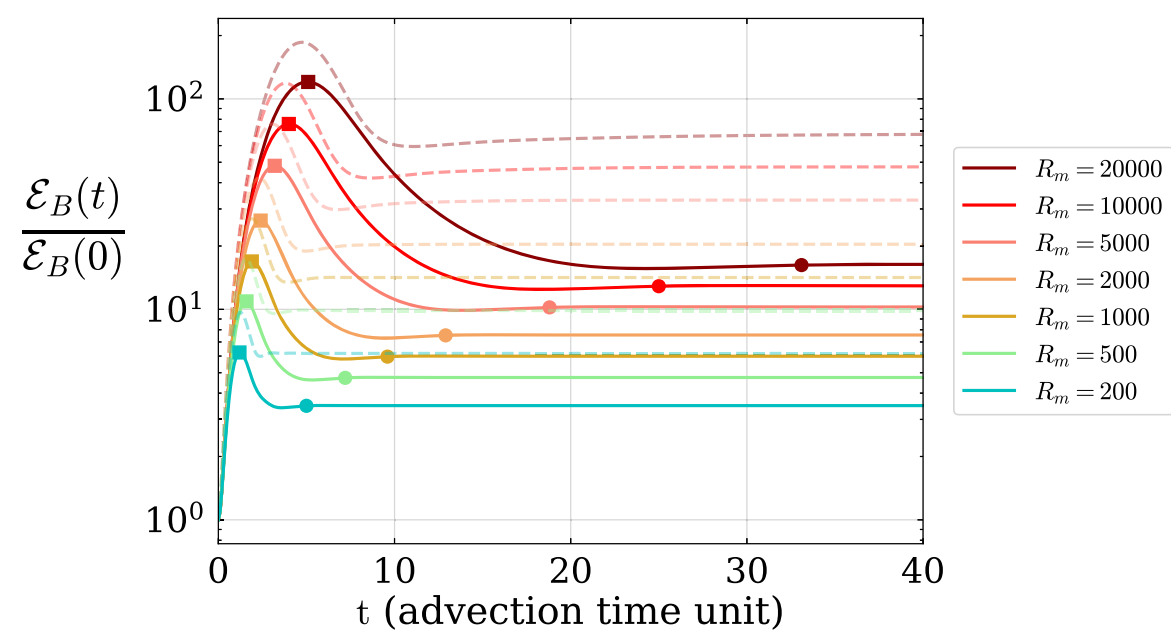

Figure 3. Time evolution of the magnetic field for Configuration 1 if the prescribed streamfunction is $\Psi_{2}$ (eq. 12). The black lines are iso-contours of the vector potential $A$, shown every $0.1 B_{0} L_{z}$. The colourscale represents the current magnetic field strength normalized by its initial value. The second row represents the magnetic field in the final stage (steady state) for a magnetic Reynolds number $R_{m}=1000$ (c) and for $R_{m}=50$ (d). (e) shows the temporal evolution of the magnetic energy normalized by its initial value for each computed $R_{m}$ for the streamfunction $\Psi_{2}$ (solid lines) compared with $\Psi_{1}$ (dashed lines). The squares (resp. circles) denote the time when the maximum (resp. steady-state) stage of the evolution is reached. See text for details. 

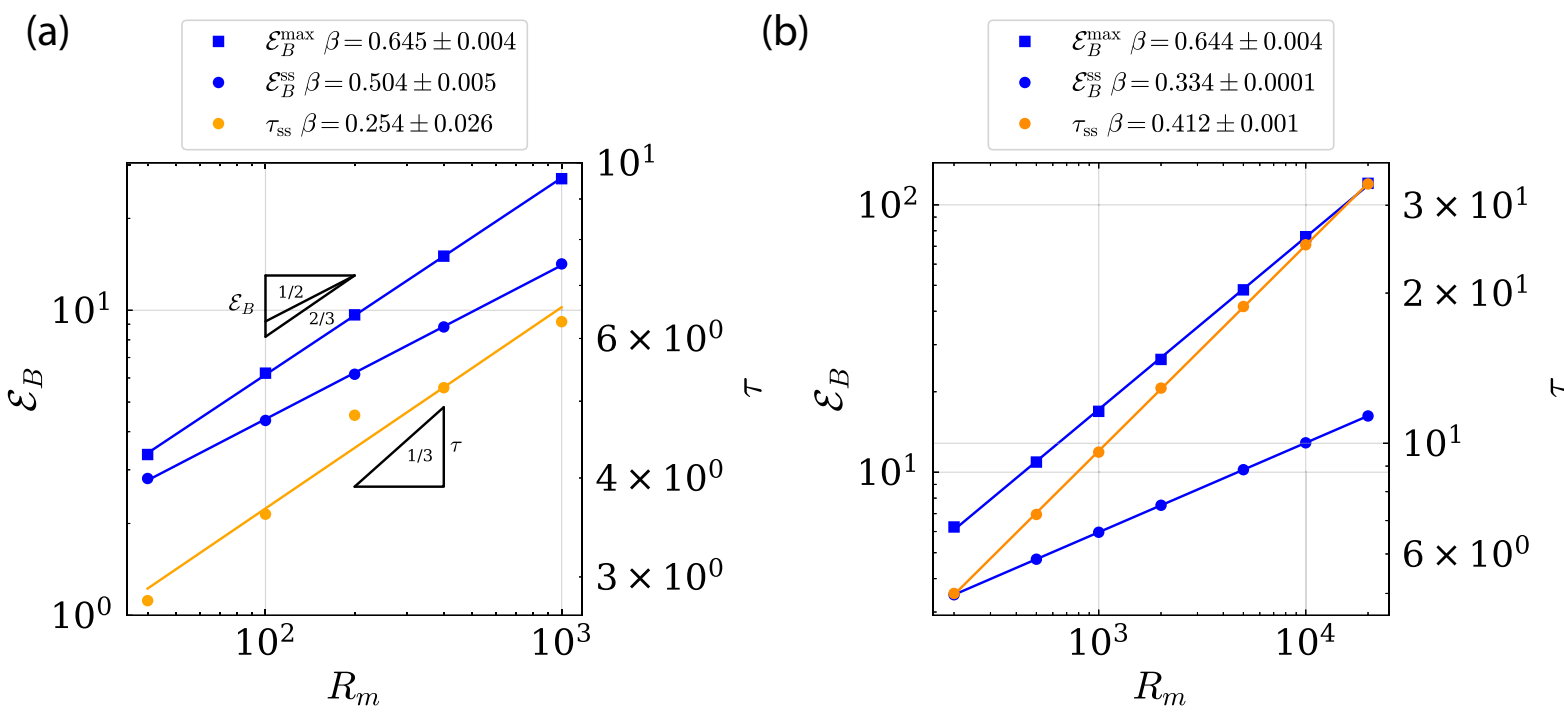

Figure 4. Dependency of the monitored quantities on the magnetic Reynolds number $R_{m}$ in Configuration 1, when the imposed streamfunction is $\Psi_{1}$ (a, see eq. 11) and $\Psi_{2}$ (b, see eq. 12). Blue squares (circles) represent the maximum (steady-state) magnetic energy $\mathcal{E}_{B}^{\max }\left(\mathcal{E}_{B}^{\text {ss }}\right)$; the time to reach steady-state energy $\tau_{\mathrm{ss}}$ is shown with orange circles. The $y$-axis on the left features the scale for magnetic energy $\mathcal{E}_{B}$, while the $y$-axis on the right that for the time $\tau$. The straight lines illustrate the corresponding scaling laws found by least-squares fitting, whose form is given in the label at the top, $\beta$ denoting the exponent found in each case. Slopes of 1/3,1/2 and 2/3 indicated for reference.

Table 1. Summary of the scaling laws determined in this study, compared with the theoretical expectations. $\mathcal{E}_{B}^{\max }$ and $\mathcal{E}_{B}^{\text {ss }}$ are the maximum energy and the energy at the steady state, respectively, with $\tau_{\max }\left(\mathcal{E}_{B}^{\max }\right)$ and $\tau_{\mathrm{ss}}$ their corresponding characteristic timescales. $\left(B_{z 0}^{\max }\right)^{\max }$ is the maximal amplitude of the $z$-component of the field at the insulating top boundary, with $\tau_{\max }\left(\left(B_{z 0}^{\max }\right)^{\max }\right)$ its associated timescale. For Configuration $2, \Psi_{1}$, the scalings indicated for the latter quantities are the scalings at the steady state, which are the same only in this case (see text for details).

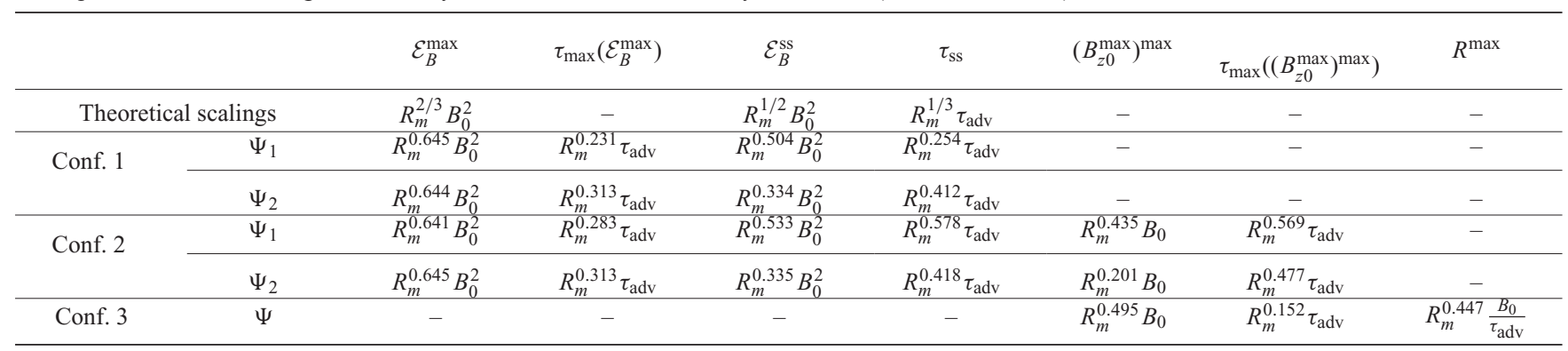

evolution that is obtained for $\Psi_{1}$ ). The time to reach the maximum amplitude is equivalent for a given $R_{m}$. As a general rule, the steady state is reached later and the corresponding amplitude of the energy is lower for $\Psi_{2}$. For example, for $R_{m}=1000$, using $\Psi_{1}$, a steady state is already reached at $t=6.5$ whereas for $\Psi_{2}, R_{m}=1000$, it is reached later than $t=8$.

The scaling laws obtained for the evolution of the magnetic energy with $\Psi_{2}$ are shown in Fig. 4(b). Note that these laws are computed for $200 \leq R_{m} \leq 2 \times 10^{5}$, and this will be the case in the remainder of this paper (in particular for the computation of the scaling laws), where we are interested in the large $R_{m}$ limit. The maximum value of the energy $\mathcal{E}_{B}^{\max }$ is found to scale as $R_{m}^{0.644 \pm 0.004}$, similar to the scale previously determined. The energy at the steady state $\mathcal{E}_{B}^{\text {ss }}$ scales as $R_{m}^{0.334 \pm 0.0001}$, much lower than previously $\left(R_{m}^{0.504}\right)$ and lower than the anticipated value of $R_{m}^{1 / 2}$. Likewise the time taken to reach the steady state is found to scale as $R_{m}^{0.412 \pm 0.001}$, as mentioned above. These differences can be ascribed to the choice of the streamfunction. $\Psi_{1}$ falls off rapidly at the upper and lower boundaries. This leads to a greater shear of the magnetic field lines close to these boundaries which in turn enhances the energy produced by the shear. Conversely, $\Psi_{2}$ produces a stronger shear close to the lateral boundaries compared to $\Psi_{1}$. The magnetic stripes are thus subject to a greater folding, subsequently leading to an increase of the relaxation time and a decrease of the total magnetic energy. As the efficiency of advection increases with the magnetic Reynolds number, the shear of the magnetic field lines becomes larger with higher $R_{m}$, thereby enhancing this relaxation. This illustrates the fact that the flow pattern (in particular the occurrence of strong flow gradients) has a strong impact on the timing and intensity of the flux expulsion mechanism.

\subsection{Configuration 2: flux expulsion from one eddy with an insulating upper boundary}

Of importance (in particular when considering geophysical/astrophysical implications) is the nature of the magnetic boundary condition that is imposed at the top of the domain. Our goal in Configuration 2 is to quantify this effect, using the previous configuration as a reference. In Configuration 2 (recall Section 2), the vertical component of the field can also diffuse through the upper boundary. Fig. 5 shows the results for the same cases as considered in the previous section (Figs 2 and 3), using $\Psi_{1}$ as our imposed 
(a)
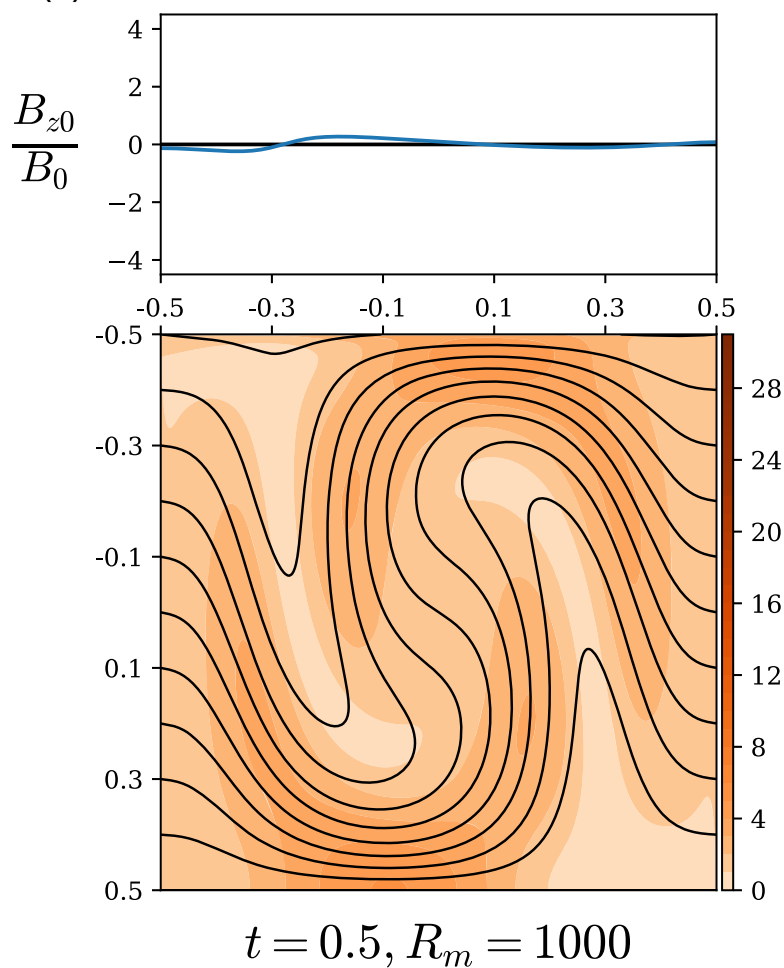

(c)
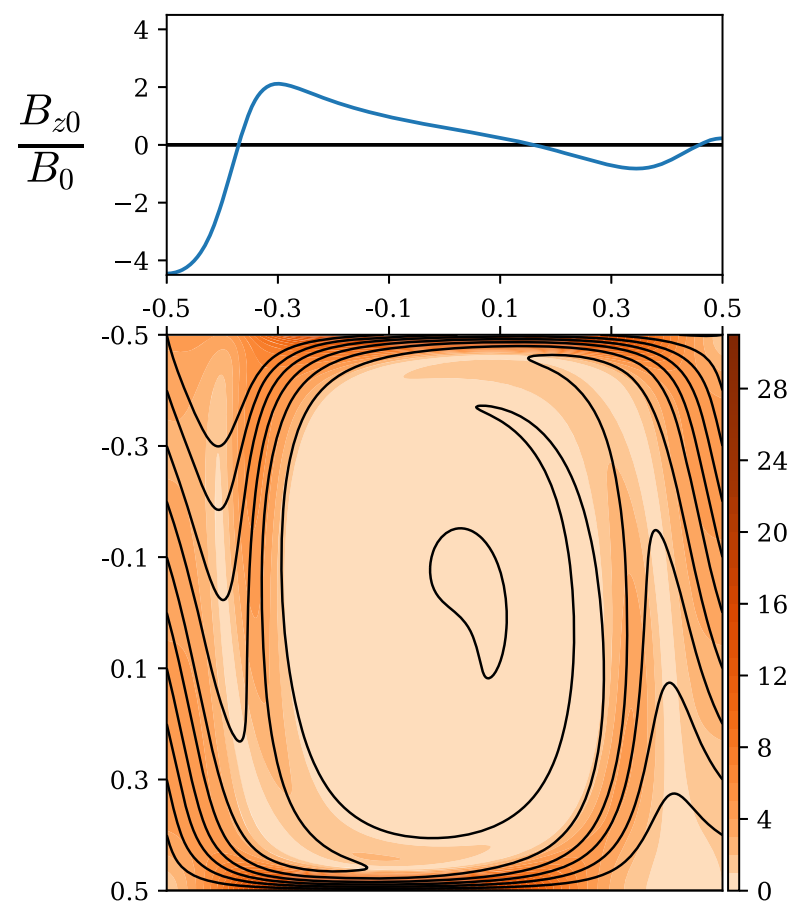

$$
t=11, R_{m}=1000
$$

(b)

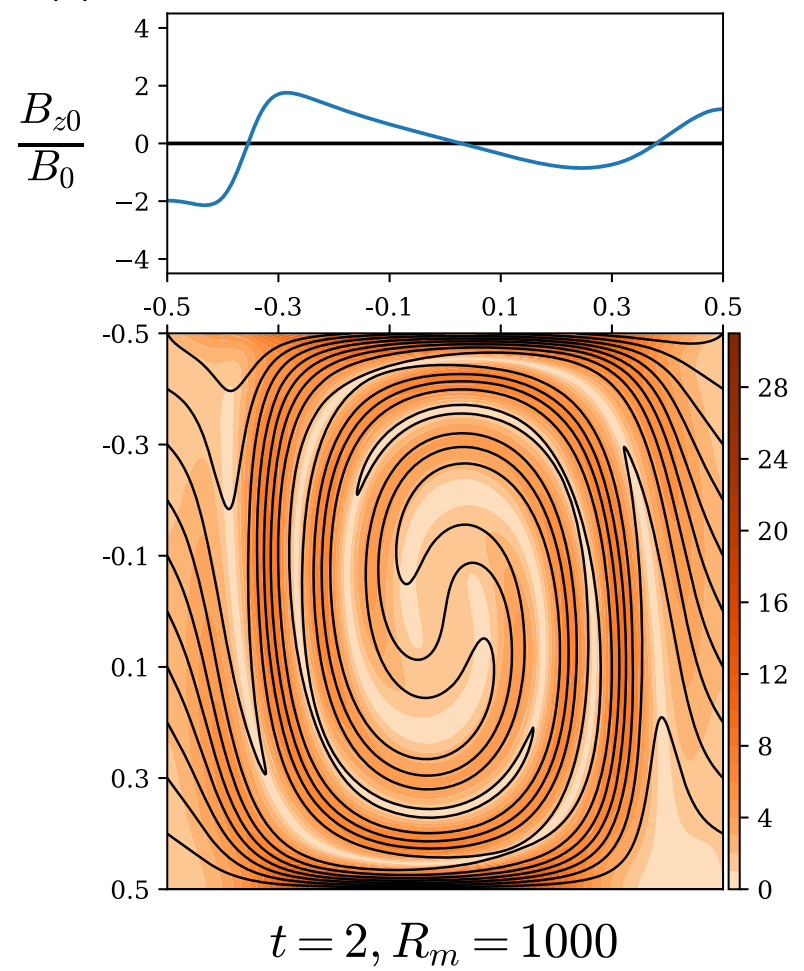

(d)
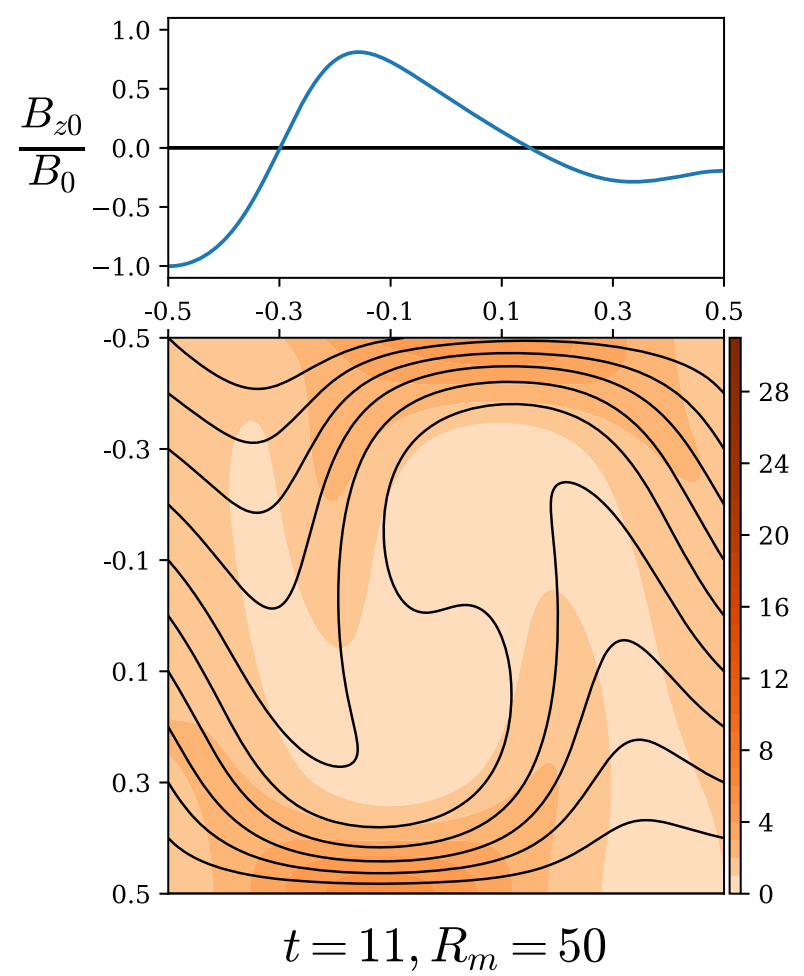

Figure 5. Time evolution of the magnetic field for Configuration 2 if the prescribed streamfunction is $\Psi_{1}$ (eq. 11). The black lines are iso-contours of the vector potential $A$, shown every $0.1 B_{0} L_{z}$. The colourscale represents the current magnetic field strength normalized by its initial value. Above each panel the blue curve shows the variation of the $z$-component of the magnetic field along the top boundary, $B_{z 0}$, at the corresponding time. The second row represents the magnetic field in the final stage (steady state) for a high magnetic Reynolds number $R_{m}$ (c) and for a low $R_{m}(\mathrm{~d})$. 
streamfunction. In the different panels of Fig. 5, each blue curve shows the vertical component of the magnetic field at the upper insulating boundary, $B_{z 0}$, as a function of $x$. For $R_{m}=1000$ (Figs 5a-c), the results are comparable with those obtained in the previous configuration (compare with Fig. 2). During the first (mainly advective) phase, magnetic field lines are twisted and folded (Fig. 5a). Then magnetic field lines start to reconnect to each other, leading to a progressive expulsion of the flux from the inside of the eddy toward its edges, which in turn leads to the increase of the amplitude of $B_{z 0}$ (Fig. 5b). The steady state is represented in Figs 5(c) and (d) for two values of $R_{m}$. For $R_{m}=1000$ (Fig. 5c), the flux is entirely expelled from the eye of the eddy at steady state. For $R_{m}=50$ (Fig. $5 \mathrm{~d}$ ), advection is not strong enough for reconnection to occur and the expulsion of the magnetic field lines from the centre of the eddy to be completed. Consequently, the amplitude of $B_{z 0}$ reached at steady state is lower in this low- $R_{m}$ case.

The same cases (same $R_{m}$ and same times for snapshots) are presented in Fig. 6 for $\Psi_{2}$. The general behaviour is similar. Note that the overall amplitude of the magnetic field intensity is lower in this case. The concentration of magnetic flux close to the upper boundary lead to an increase of the amplitude of the $z$-component of the field in $z=0$. The maximum amplitude reached at the top and bottom is lower in this case, leading to lower values of $B_{z 0}$. Another difference due to the geometry of the flow is that more flux is concentrated close to the lateral boundaries.

Fig. 7(a) shows the evolution of the energy for $\Psi_{1}$ (dashed lines) and for $\Psi_{2}$ (solid lines) for different $R_{m}$. Again, the amplitude of the energy is higher with $\Psi_{1}$. The same behaviour as described in the previous section (Figs 2 and 3) is observed. The first stage involves an increase of the energy corresponding to the advection phase. Then diffusion starts to be significant and the energy reaches a maximum and decreases, as magnetic field lines reconnect to each other, until all the flux is expelled from the eye, and steady state is reached. The scaling laws (Figs $7 \mathrm{~b}$ and c, summarized in Table 1) obtained to characterize the energy at its maximum and at steady state are close to those obtained for the previous configuration. $\mathcal{E}_{B}^{\max }$ is found to scale as $R_{m}^{0.641 \pm 0.001}$ and as $R_{m}^{0.645 \pm 0.004}$ for $\Psi_{1}$ and $\Psi_{2}$, respectively (exponents equal to 0.645 and 0.644 resp. for Configuration 1). In the first configuration, energy amplitudes are slightly higher; the scaling law shows that there is no difference regarding the trend of the evolution. $\mathcal{E}_{B}^{\text {ss }}$ is found to scale as $R_{m}^{0.533 \pm 0.003}$ and $R_{m}^{0.335 \pm 0.0002}$ for $\Psi_{1}$ and $\Psi_{2}$, respectively, similar to Configuration 1 in both cases $(0.504$ and 0.334 , resp.). The time to reach the steady state scales also as in Configuration 1 for $\Psi_{2}$ : the exponent is 0.418 \pm 0.001 (against 0.412). It is rather different for $\Psi_{1}: 0.578 \pm 0.030$ (against 0.254). These results show that the effect of the insulating boundary on the energy variations is limited. However, it has a noticeable effect on the characterization of the steady state. The diffusion of the flux through the insulating boundary takes longer than the expulsion of the flux from the centre of the eddy which leads to longer relaxation time at higher $R_{m}$.

The same systematic analysis is applied to the evolution of $B_{z 0}^{\max }$ (Fig. 8). Fig. 8(a) shows the evolution with time of $B_{z 0}^{\max }$ for $\Psi_{1}$ (dashed lines) and for $\Psi_{2}$ (solid lines). It is striking that for $\Psi_{1}$, the amplitude of $B_{z 0}^{\max }$ is significantly higher than that obtained with $\Psi_{2}$. For the latter, $B_{z 0}^{\max }$ grows rather slowly until reaching a maximum, then it decreases to the steady state value (see the solid curves in Fig. 8). In contrast, for $\Psi_{1}, B_{z 0}^{\max }$ grows faster (over a longer time than for $\Psi_{2}$, several tens of advection times for the largest values of $\left.R_{m}\right)$, until it plateaus at a maximum value $\left(B_{z 0}^{\max }\right)^{\max }$ coinciding with the steady state. The resulting scaling laws (see Table 1$)$ are represented in Figs 8 (b) and (c) for $\Psi_{1}$ and $\Psi_{2}$, respectively. $\left(B_{z 0}^{\max }\right)^{\max }$ is found to scale as $R_{m}^{0.201 \pm 0.009}$ (for $\Psi_{2}$ ). The threshold value $C_{2}$ used to define the steady state (recall eq. 8) is chosen as $C_{2}=$ $5 \times 10^{-4}$ for $\Psi_{1}$ and $C_{2}=5 \times 10^{-5}$ for $\Psi_{2}$. At steady state, $\left(B_{z 0}^{\max }\right)^{\text {ss }}$ scales as $R_{m}^{0.435 \pm 0.007}$ and $R_{m}^{0.237 \pm 0.003}$ for $\Psi_{1}$ and $\Psi_{2}$, respectively. The time to reach the steady state is found to scale as $R_{m}^{0.569 \pm 0.007}$ and $R_{m}^{0.425 \pm 0.012}$ for $\Psi_{1}$ and $\Psi_{2}$, respectively. The different results for the different streamfunctions are again a consequence of the behaviour of the different flows at the boundaries. The larger flow gradients at the top and bottom boundaries lead to a greater energy concentration for $\Psi_{1}$, particularly at the top: this enhances the amplitude of $B_{z 0}^{\max }$. Conversely, the slightly stronger flow gradients on the lateral boundaries for $\Psi_{2}$ generate a stronger destructive folding of magnetic fields lines on those boundaries, and consequently to a decrease of $B_{z 0}^{\max }$. We do not observe this behaviour if $\Psi_{1}$ is prescribed. These results show that $\Psi_{1}$ is more efficient for the expulsion of flux from the eddy and through the insulating upper boundary. We ascribe its efficiency to its geometrical properties. As described in the previous configuration, strong flow gradients enhance the magnetic energy at the boundary. Here, gradients in both $z$ and $x$ are important: the former allows the flux to concentrate in the vicinity of the boundary, while the latter concentrates the flux near downwellings, thereby enabling an amplification of $B_{z 0}$.

In this configuration, the evolution of $\left(B_{z 0}^{\max }\right)^{\max }$ is primarily controlled by the lateral boundary conditions. The magnetic field lines being fixed on lateral boundaries, they are driven downward by the downwelling on the left boundary which increases the $z$-component of the field in $x=0$. $\left(B_{z 0}^{\max }\right)^{\max }$ is thus located at $x=0$, where the magnetic field lines are fixed.

\subsection{Configuration 3: flux expulsion from two eddies with an insulating upper boundary}

The third configuration retains the same top and bottom boundary conditions for the vector potential as those for Configuration 2, with an upper insulating boundary and a perfectly conducting bottom boundary. As shown in Fig. 1c), the domain $\mathcal{D}$ is rectangular with a 2:1 aspect ratio $\left(L_{z}=1, L_{x}=2\right)$, and the fluid is set in motion by two eddies producing a convective upwelling at the centre of the domain. This flow is defined by the streamfunction

$\Psi(x, z)=\frac{1}{\pi} \sin (\pi x) \sin (\pi z)$.

This streamfunction has the same behaviour as $\Psi_{1}$ along the $z$ direction and should therefore maximize concentration of flux near the top boundary. The initial magnetic field is again horizontal (see the blue arrows in Fig. 1c). A noticeable difference with regard to Configurations 1 and 2 is that the magnetic field lines are no longer tied to the lateral sides, as was the case in the one-vortex canonical configuration, in order to reproduce exactly the configuration used by Bloxham (1986). This choice is further motivated by the fact that it is not relevant to fix the field lines at the lateral boundaries as they are supposed to reflect the behaviour of the toroidal magnetic field in the Earth's outer core.

Results are presented in Fig. 9 for $R_{m}=1000$ at $t=1.5$ (Fig. 9.a), $t=3.5$ (Fig. 9.b) and $t=30$ (Fig. 9.c). As previously seen in the single-eddy configurations for high $R_{m}$ cases, the magnetic field lines initially inside the eddies are at first wound and concentrated near the edges of the domain (Fig. 9a, bottom panel). Reconnection of field lines occurs when two stripes of opposite polarity are close enough (Fig. 9b, bottom panel); this leads to the progressive expulsion of magnetic flux from the interior of each eddy to its edges. The evolution of $B_{z 0}$ (top panels of Figs $9 \mathrm{a}-\mathrm{c}$ ) is however different 
(a)

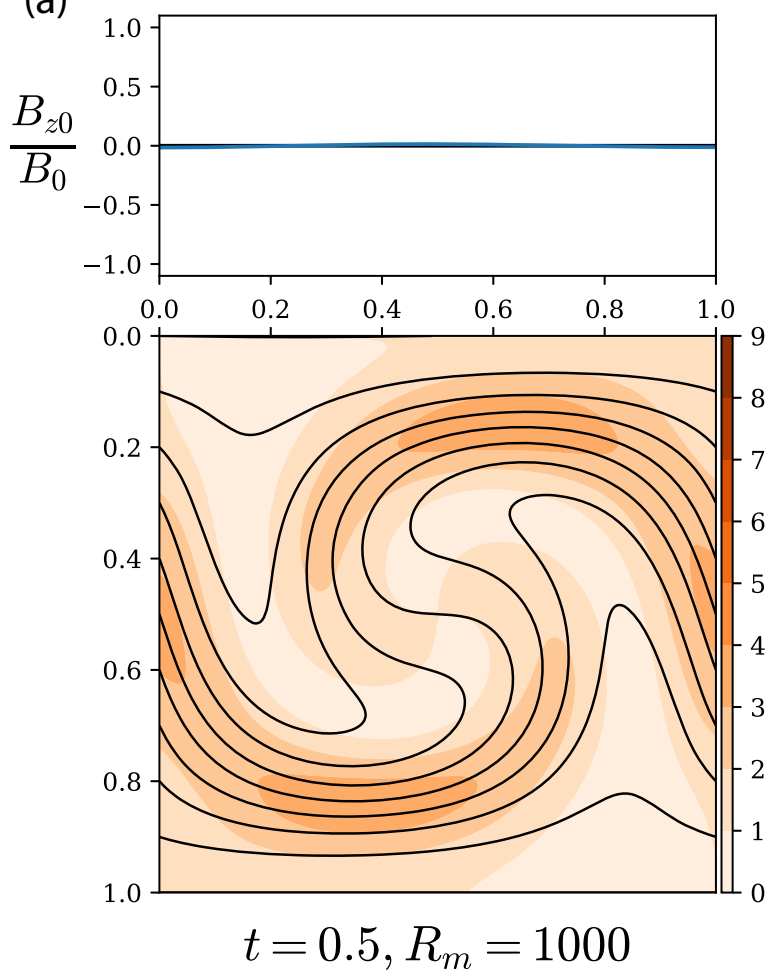

(c)
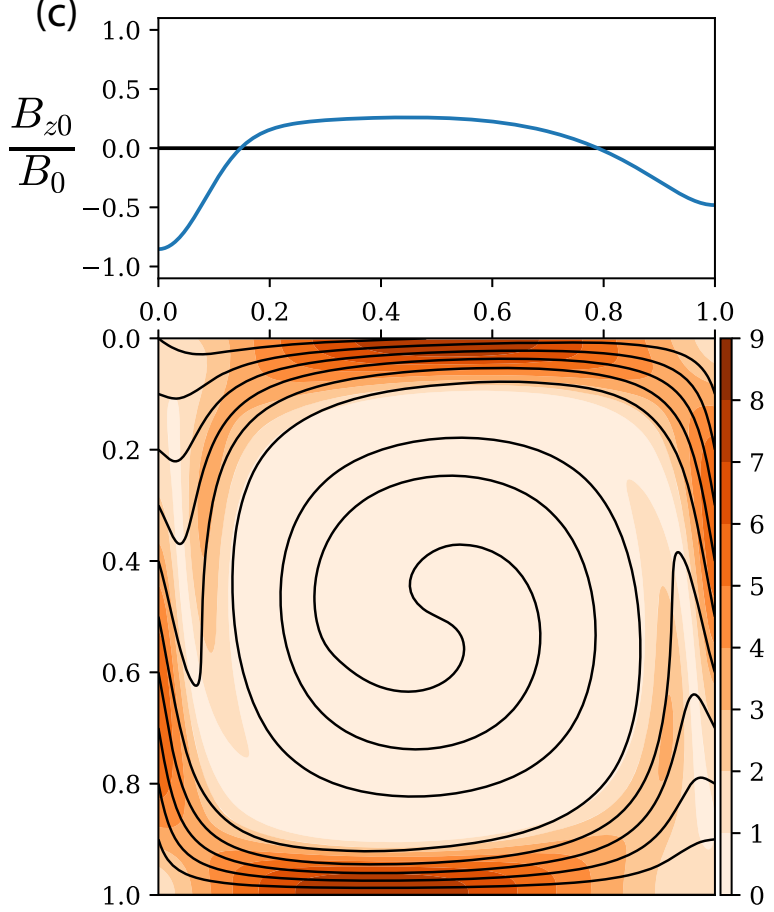

$t=11, R_{m}=1000$ (b)
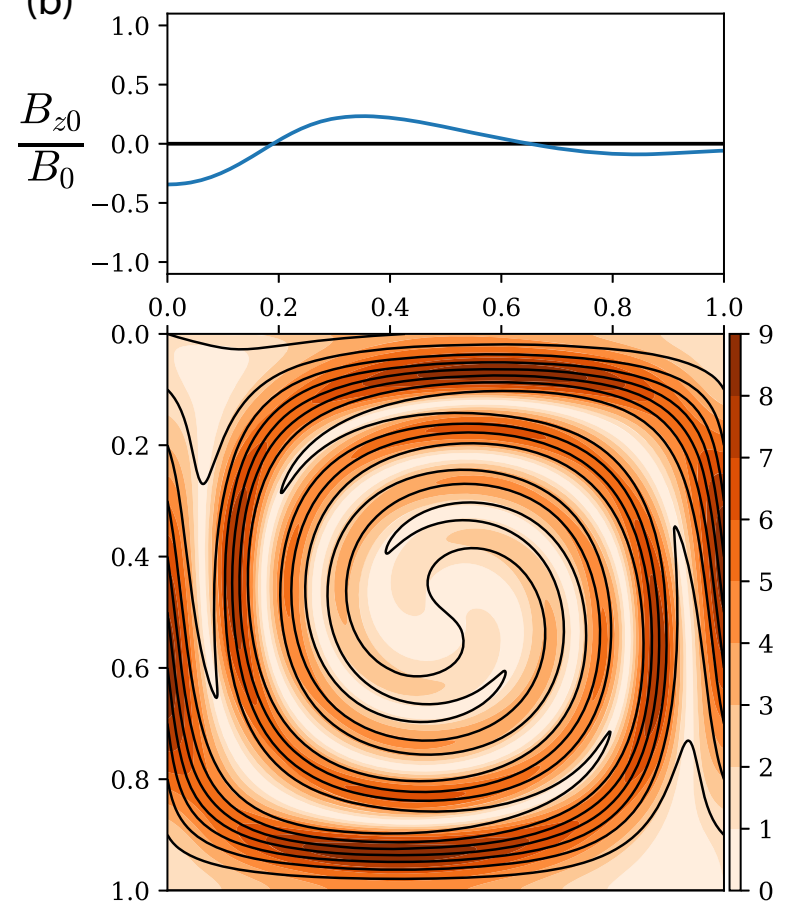

$t=2, R_{m}=1000$

(d)
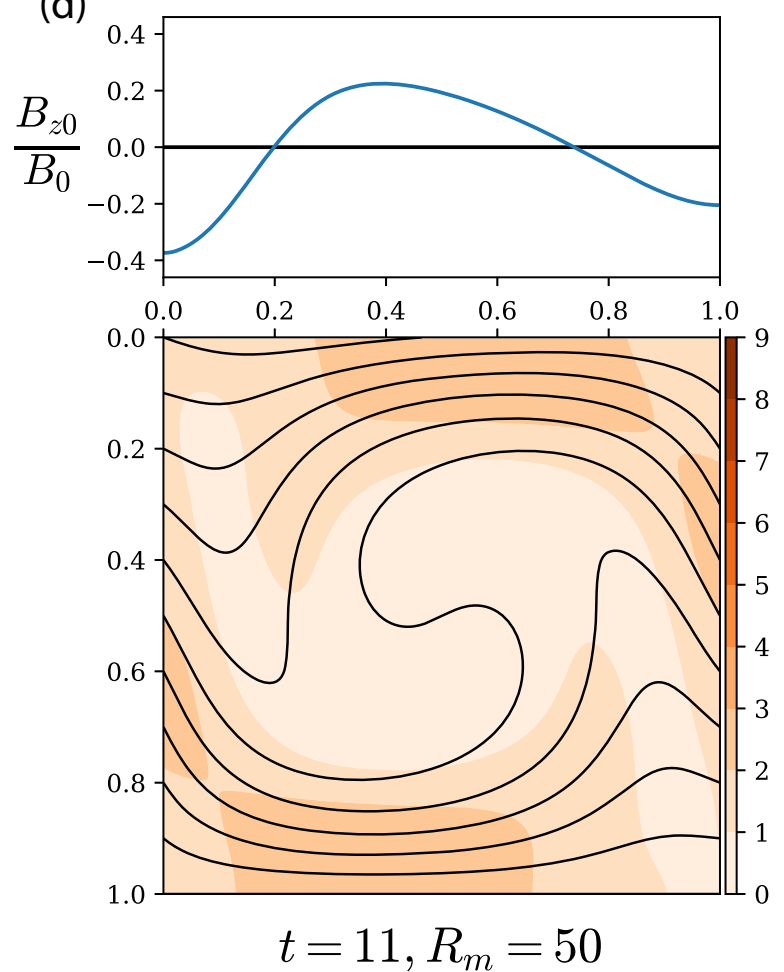

Figure 6. Time evolution of the magnetic field for Configuration 2 if the prescribed streamfunction is $\Psi_{2}$ (eq. 12). The black lines are iso-contours of the vector potential $A$, shown every $0.1 B_{0} L_{z}$. The colourscale represents the current magnetic field strength normalized by its initial value. Above each panel, the blue curve shows the variation of the $z$-component of the magnetic field along the top boundary, $B_{z 0}$, at the corresponding time. The second row represents the magnetic field in the final stage (steady state) for a magnetic Reynolds number $R_{m}=1000$ (c) and for $R_{m}=50$ (d). 
(a)

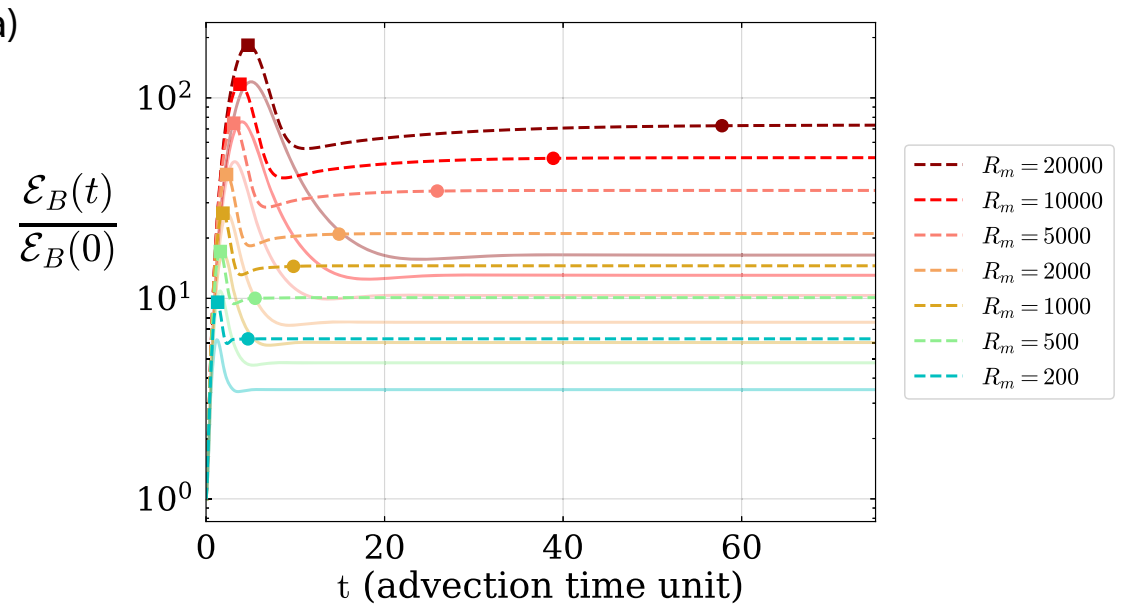

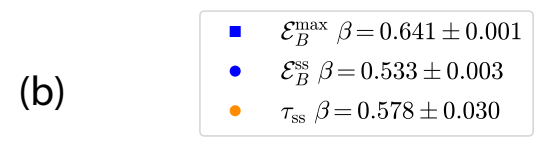

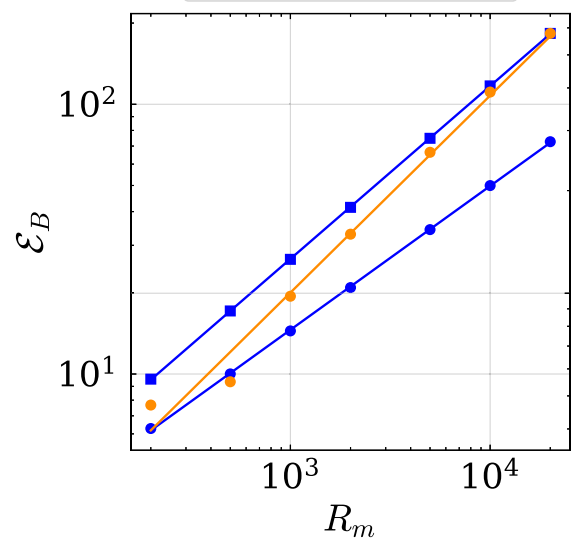

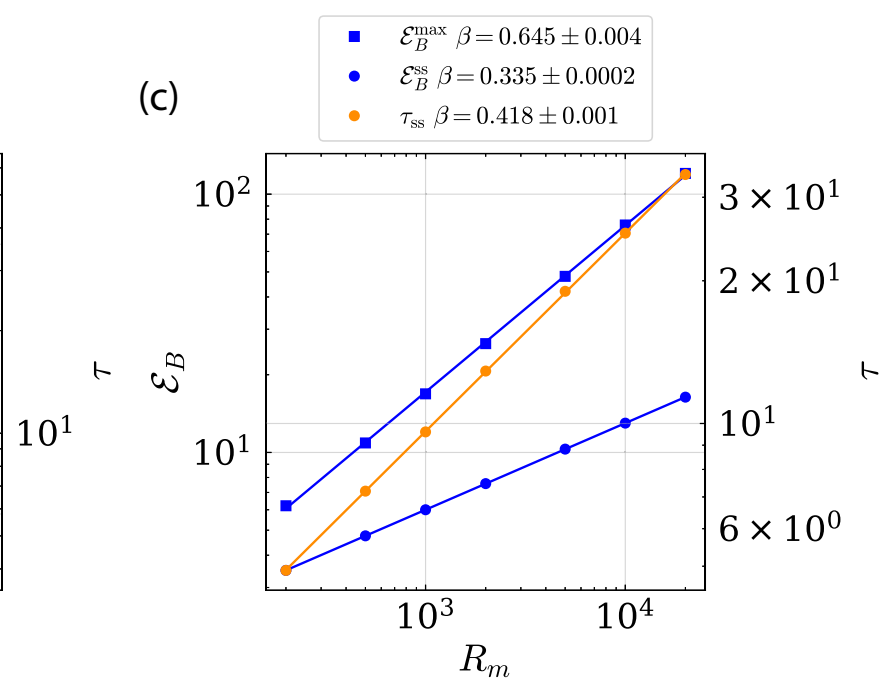

Figure 7. Evolution of the total magnetic energy for Configuration 2. (a): evolution of the magnetic energy with time for a range of magnetic Reynolds number $R_{m}$ in the case of the streamfunction $\Psi_{1}$ (dashed lines) and $\Psi_{2}$ (solid lines). (b) and (c): evolution of the maximum of the magnetic energy $\mathcal{E}_{B}^{\text {max }}$, its amplitude at the steady state $\mathcal{E}_{B}^{\text {ss }}$, and their corresponding characteristic times, $\tau_{\max }$ and $\tau_{\mathrm{ss}} \tau_{\mathrm{ss}}$, respectively, as a function of $R_{m}$ (ranging from 200 to 20000 ), for $\Psi_{1}$ and $\Psi_{2}$, respectively. The straight lines illustrate the corresponding scaling laws found by least-squares fitting, whose form is given in the label at the top, $\beta$ denoting the exponent found in each case.

from that observed in Configuration 2: it is now antisymmetric with respect to the centre of the upwelling, with two intensity peaks of opposite signs and same amplitude, located above the downwellings. As the flux is further concentrated near the top left and right corners of the domain, the amplitude of the peaks of $B_{z 0}$ increases with time (Fig. 9b), until it finally reaches a steady state (Fig. 9c).

The behaviour of the peak amplitude, $B_{z 0}^{\max }$, normalized by the initial magnetic field strength $B_{0}$, is documented as a function of time for different magnetic Reynolds numbers in Fig. 10 (left-hand panel). Note that the integration times necessary to reach a steady state at large $R_{m}$ are substantially longer (several tens of $\tau_{\text {adv }}$ ) than those considered by Bloxham (1986), whose range of $R_{m}$ was restricted between 10 and 200. When compared with Configuration 2, the same increase is seen at first, over a few $\tau_{\text {adv }}$, following the initial shear-augmented diffusion of flux. At any given $R_{m}$, the maximum amplitude that is reached is however larger than the one obtained for Configuration 2 (Fig. 8a). The evolution of $B_{z 0}^{\max }$ is not controlled by the lateral boundary conditions (as in Configuration 2), but by the flow gradients in $x$ concentrating the flux near downwellings. The magnetic field lines move freely along the lateral boundaries, so the flow drags them downward and no vertical component is created as the magnetic field lines are not distorted. Once the maximum (of $\left.B_{z 0}^{\max }\right)$ is obtained at time $t=\tau_{\max }$, relaxation to steady state occurs. This relaxation can be oscillatory in nature, and its amplitude increases with $R_{m}$. We interpret these oscillations as originating from the arrival near the top of the domain of the magnetic field stripes of alternating polarity created by the distortion of magnetic field lines and their subsequent diffusion. Such oscillations do not occur in the case of Configuration 2 precisely because the evolution of $B_{z 0}^{\max }$ is in this case governed by field lines being tied to the lateral boundaries. In addition, note that these oscillations can exist by virtue of the 2-D geometry. Flux can not escape in the third direction of space. In three dimensions, for instance in the case of a helical upwelling flow, the situation may be different.

Our systematic survey of $R_{m}$ values allows us to derive scaling laws describing the evolution of $B_{z 0}^{\max }$ (see Table 1). To define the steady state, we used a threshold value $C_{2}=5 \times 10^{-3}$ (recall eq. 8). As shown in Fig. 10(right-hand panel), we find that

$$
\begin{aligned}
\tau_{\max } & =1.002 \pm 0.012 R_{m}^{0.152 \pm 0.004}, \\
\left(B_{z 0}^{\max }\right)^{\max } / B_{0} & =0.267 \pm 0.019 R_{m}^{0.495 \pm 0.006}
\end{aligned}
$$


(a)

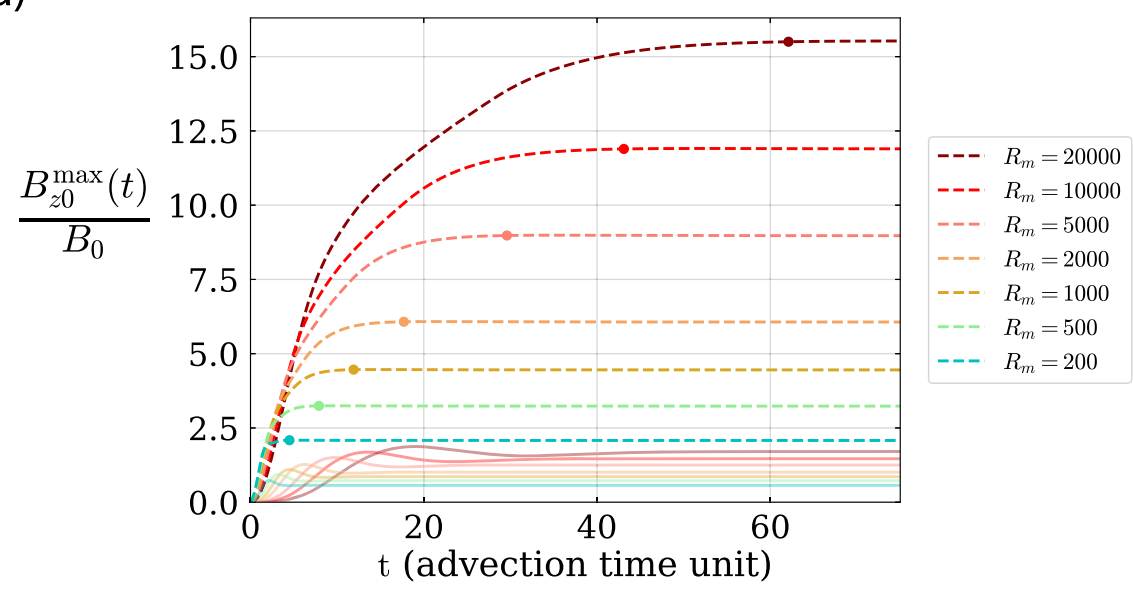

(b)
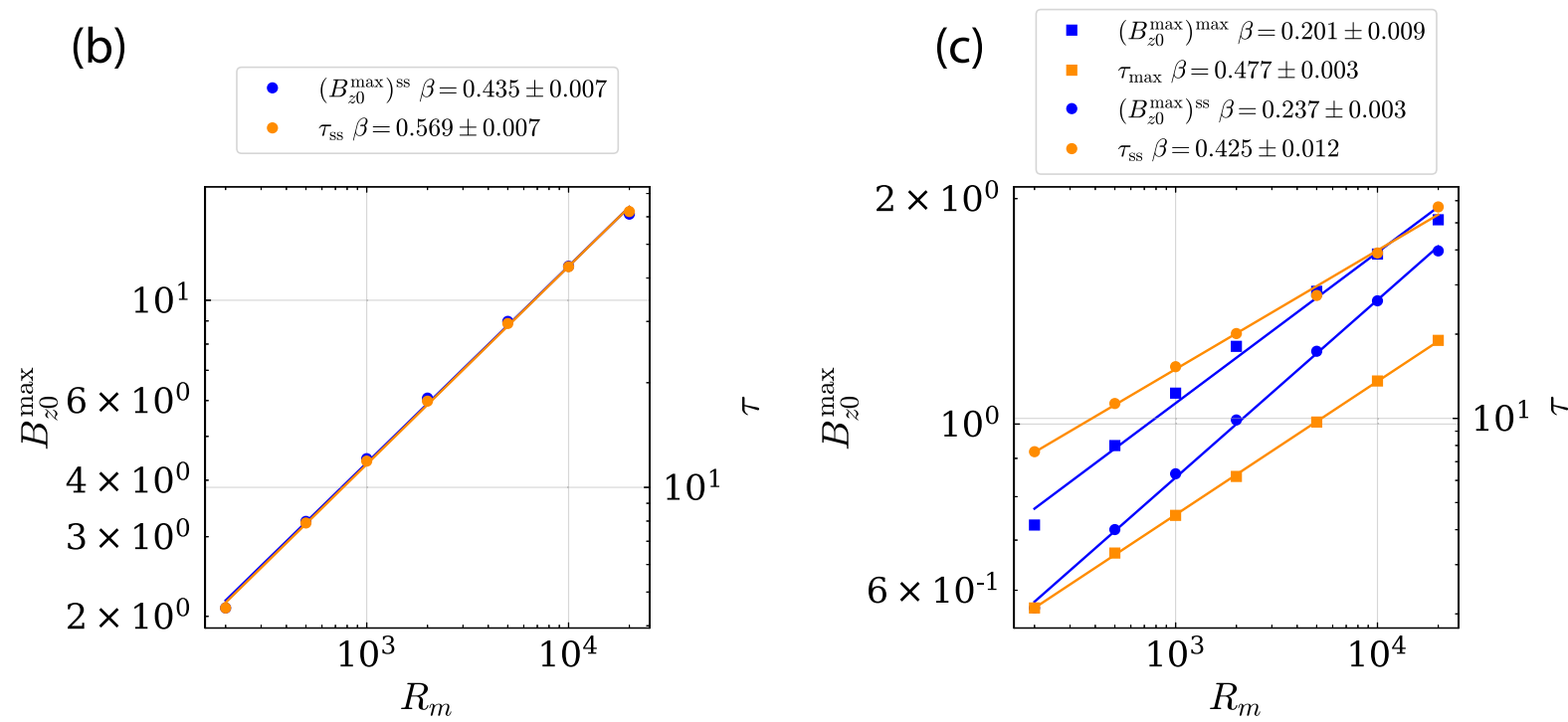

Figure 8. Evolution of the maximum of the $z$-component of the magnetic field at the top insulating boundary, $B_{z 0}^{\max }$ (eq. 6), for Configuration 2. (a) shows the evolution of $B_{z 0}^{\max }$ with time for a range of magnetic Reynolds number $R_{m}$ for the streamfunction $\Psi_{1}$ (dashed lines) and $\Psi_{2}$ (solid lines). (b) and (c) show the evolution of the monitored quantities as a function of $R_{m}$ together with the associated scales for $\Psi_{1}$ and $\Psi_{2}$, respectively. The straight lines illustrate the corresponding scaling laws found by least-squares fitting, whose form is given in the label at the top, $\beta$ denoting the exponent found in each case.

provide an adequate least-squares fit (in log-log space) to the data. Restoring dimensions, these scaling laws become

$$
\begin{aligned}
\tau_{\max } & =1.002 \frac{L}{U}\left(\frac{U L}{\eta}\right)^{0.152}, \\
\left(B_{z 0}^{\max }\right)^{\max } & =0.267 B_{0}\left(\frac{U L}{\eta}\right)^{0.495} .
\end{aligned}
$$

To reiterate, the pseudo-convective flow prescribed here causes a more intense flux concentration near the top left- and right-hand corners of the domain, regardless of the value of $R_{m}$.

The increase of $B_{z 0}^{\max }$ between the initial state at $t=0$ and the maximum at $t=\tau_{\max }$ can be averaged, under the assumption of a linear increase. The ratio of $\left(B_{z 0}^{\max }\right)^{\max }$ to $\tau_{\max }$ therefore gives an estimate of this average variation rate and its dependency on $R_{m}$. We find that this average variation rate scales as $\sim R_{m}^{0.343} B_{0} U / L$. However, as shown by the evolution of $B_{z 0}^{\max }$ (recall Fig. 10, lefthand panel), the instantaneous rate of change $R$ (eq. 9) can be faster than this average at some instants. We determine the scale for the maximum instantaneous rate of change (defined by eq. 10) by a systematic survey of this quantity for the same range of $R_{m}$. We find a stronger dependency on $R_{m}$ (with an exponent equal to 0.447 ) which yields the following dimensional law

$$
R^{\max }=0.300 B_{0} \frac{U}{L}\left(\frac{U L}{\eta}\right)^{0.447} \text {. }
$$

\section{DISCUSSION}

In order to provide plausible estimates for magnetic field variations that could be produced by flux expulsion from the Earth's core, we base the discussion on results obtained from Configuration 3 . Although simplified, this configuration appears to be the most efficient at generating flux expulsion through the upper boundary. In addition, the specific geometry and fluid flow pattern imposed by Bloxham (1986) produces a pair of peaks with opposite sign obtained at the top insulating boundary (taking the periodicity of the solution into account, recall Fig. 9) which is reminiscent of pairs of flux patches of opposite polarity seen at the top of the core (see for example Jackson \& Finlay 2015). 
(a)

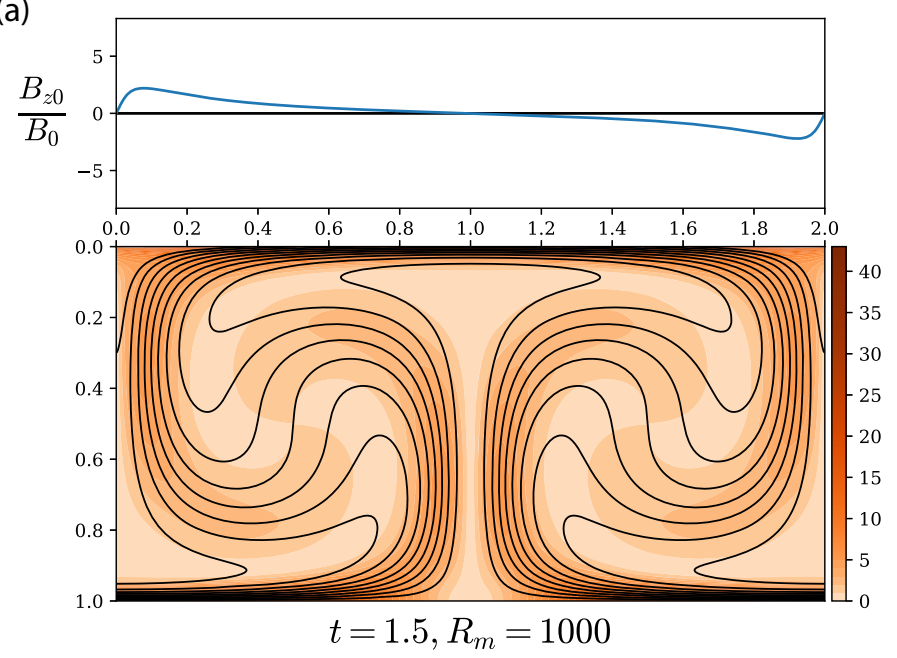

(b)

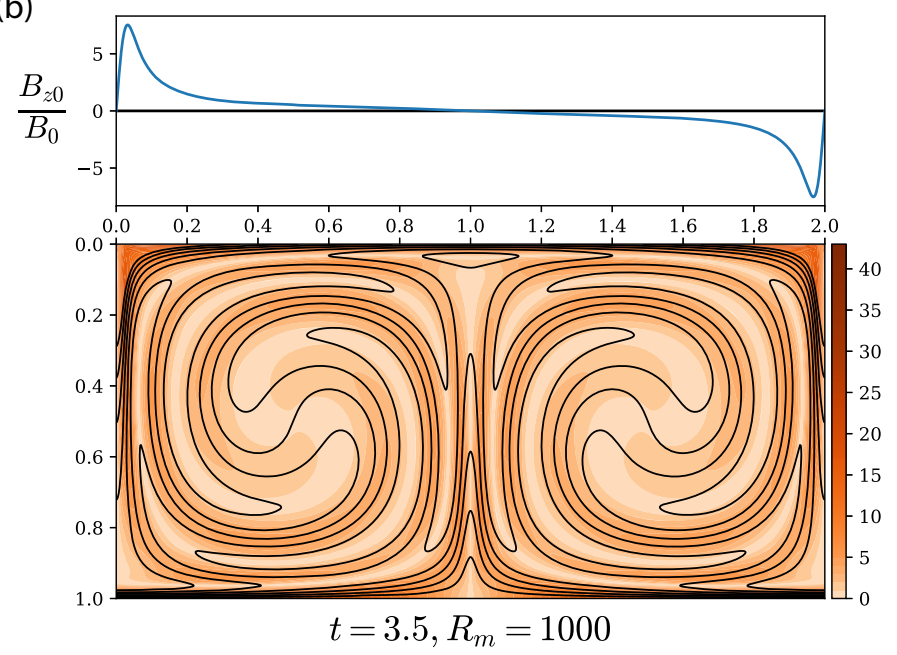

(c)

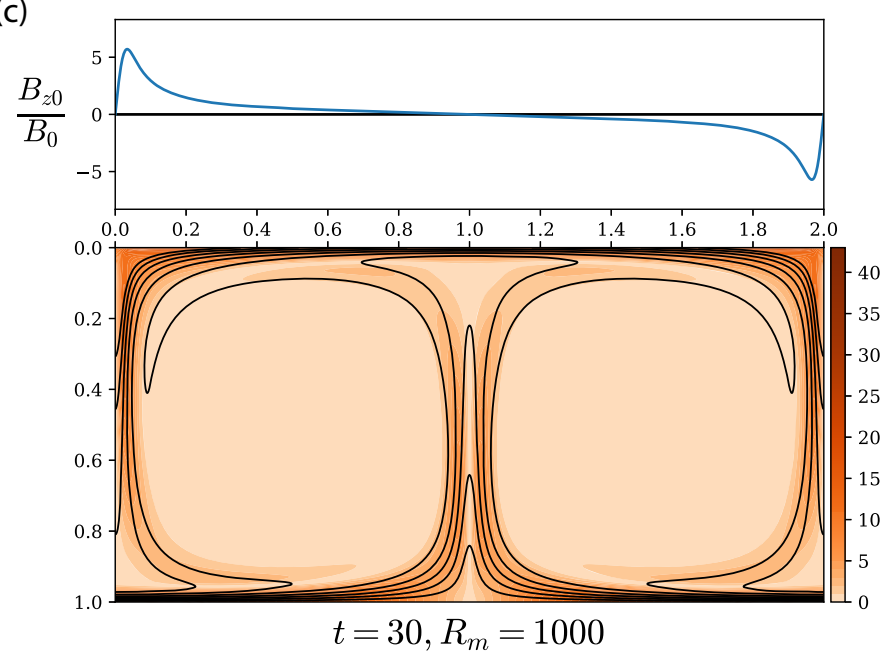

Figure 9. Time evolution of the magnetic field for Configuration 3 if the prescribed streamfunction is $\Psi$ (eq. 13), for a magnetic Reynolds number $R_{m}=1000$. The black lines are iso-contours of the vector potential $A$, shown every $0.1 B_{0} L_{z}$. The colourscale represents the current magnetic field strength normalized by its initial value. Above each panel the blue curve shows the variation of the $z$-component of the magnetic field along the top boundary, $B_{z 0}$. 

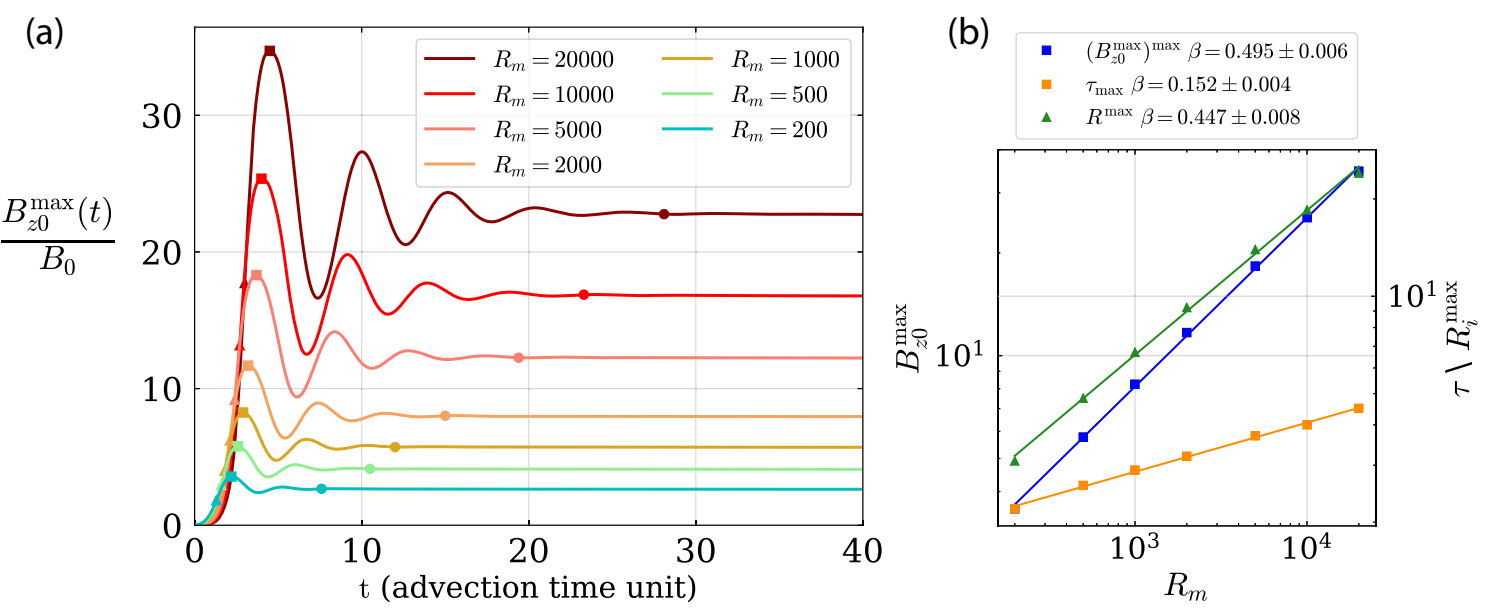

Figure 10. (a) Evolution of the maximum of the $z$-component of the field at $z=0, B_{z 0}^{\max }$ (eq. 6), for a range of magnetic Reynolds number $R_{m}$. (b) Shows the evolution of the monitored quantities as a function of $R_{m}$ together with the associated scales. The straight lines illustrate the corresponding scaling laws found by least-squares fitting, whose form is given in the label at the top, $\beta$ denoting the exponent found in each case.

Configuration 3 is however based on a number of assumptions, the consequences of which need to be discussed. First, we consider a 2-D domain. As mentioned in the previous section, the same evolution for $\left(B_{z 0}^{\max }\right)^{\max }(t)$ is not expected in 3-D and the oscillations observed before the steady-state is reached may disappear. In their study, Galloway et al. (1978) argued that in 3-D, in the large $R_{m}$ limit, the flow concentrates the flux in a rope, amplifying the field inside as a function of $\sim R_{m} B_{0}$, instead of $\sim R_{m}^{1 / 2} B_{0}$ obtained in the 2-D, sheet-like configuration. A fully $3-\mathrm{D}$ geometry therefore increases $\left(B_{z 0}^{\max }\right)^{\max }$, since it increases the concentration of magnetic flux. The work by Galloway et al. (1978) focuses on the solar dynamo, which is not dramatically influenced by the background rotation of the Sun. On Earth, however, rotational effects are of foremost importance, and the Coriolis force imparts an invariance of the flow in the direction of Earth's background rotation, making the flow 2-D in practice. Our 2-D model could in this respect represent the situation occurring in the equatorial plane (or any plane parallel to the equatorial plane), where two counter-rotating eddies may contribute to the expulsion of magnetic flux. Secondly, we consider two perfectly symmetric counter-rotating eddies. If this symmetry were broken, the $z$-component of the magnetic field at the upper insulating boundary would no longer be symmetrical, as the shear of the magnetic field lines by the two eddies would no longer be the same. The stronger eddy would give rise to a stronger flux expelled through the boundary. $\left(B_{z 0}^{\max }\right)^{\max }$ would still depend on the streamfunction, in particular on the gradient that controls the shear of the magnetic field lines. Thirdly, we consider an initial magnetic field that is horizontal and uniform. Were this initial field not uniform, in the high $R_{m}$ limit, magnetic field lines would still be rapidly swept aside and concentrated at the boundaries. We therefore do not expect a significant change in the scaling laws from a change in the initial magnetic configuration (provided that the average magnetic field strength remains the same). Fourthly, the steady flow considered here is extremely efficient in concentrating and shearing magnetic field lines in the vicinity of the upper boundary. In the case of a non-steady flow, this optimal geometry would not be sustained over the time span it takes to generate the expulsion of flux. This would lower the efficiency of the process. We thus expect $\left(B_{z 0}^{\max }\right)^{\max }$ to be either lower and/or achieved over a longer duration $\tau_{\max }$. Finally, it is also important to stress that this 2-D model is kinematic and does not account for the Lorentz force: the above rates overestimate the rate of flux expulsion. In a dynamic context, the Lorentz force is likely to exert a feedback on the fluid flow, in particular in the case of a strong toroidal field. Magnetic tension decreases the flow speed, which is detrimental to both the amount of flux advected towards the boundary and the magnitude of the field gradients. In spite of these intrinsic limitations, we think that Configuration 3 gives relevant information to understand whether flux expulsion events can generate geomagnetic spikes as documented in the recent literature.

The extrapolation of our deduced scaling laws (eq. 15) to the Earth requires constraints on the fluid velocity and on the magnetic diffusivity at the $\mathrm{CMB}$, as well as on the intensity of the toroidal magnetic field close to the CMB and the typical lengthscale of the convective process. Reasonable estimates are available at least for the two first parameters. The rms velocity of the fluid at the $\mathrm{CMB}$ is $\sim 15 \mathrm{~km} \mathrm{yr}^{-1}$ (Jones 2015), a value derived from the study of the magnetic flux patches advection at the CMB. The magnetic diffusivity is also usually assumed to take a value close to $\eta \sim$ $0.6 \mathrm{~m}^{2} \mathrm{~s}^{-1}$ (Pozzo et al. 2012). Below we discuss the impact of other, more speculative, choices for these parameters.

Constraining the two remaining parameters is more challenging. The degree 13 of spherical harmonic decomposition is the smallest wavelength of the contribution of the core field that is observable at the Earth's surface, the crustal magnetic field hiding its smaller wavelength contribution. Truncation at degree 13 is a common practice used for core field models, for instance those contributing to the IGRF (Thébault et al. 2015). The corresponding minimum distance $L$ at the CMB is then $\sim 800 \mathrm{~km}$. Adopting $U=15 \mathrm{~km} \mathrm{yr}^{-1}, \eta=$ $0.6 \mathrm{~m}^{2} \mathrm{~s}^{-1}, L=800 \mathrm{~km}$ leads to a local $R_{m} \sim 630$. Application of our scaling laws characterizing flux expulsion then gives a duration of $\tau_{\max } \sim 140 \mathrm{yr}$ associated with a total duration of $\tau_{\text {tot }} \sim 280 \mathrm{yr}$ accounting for an increase and a decrease of the intensity (to obtain a peak, $c f$. Table 2). Based on these estimates, $140 \mathrm{yr}$ is therefore the shortest timescale that may be detected at the Earth's surface due to flux expulsion at the CMB. On the other hand, one can instead take as the relevant lengthscale the thickness of the outer core, that is $L \sim 2 \times 10^{3} \mathrm{~km}$, which corresponds to spherical harmonic degree $\sim 5$ at the Earth's surface. This leads to a local $R_{m} \sim 1590$ and a duration $\tau_{\max } \sim 410 \mathrm{yr}$, leading to a total duration $\tau_{\text {tot }} \gtrsim 820 \mathrm{yr}$.

Turning to the toroidal field confined within the Earth's core, it is clear that determining its intensity is far from trivial. Hori et al. (2015) suggest a lower bound of $3 \mathrm{mT}$ from the analysis of the 
Table 2. Extrapolation of the scaling laws given by eqs (15) and (16) at the CMB and at the Earth's surface for various choices of characteristic scales. The last two columns give variation rates at the CMB and at the Earth's surface respectively. These rates are comprised between the average variation rate (lower value) and the maximum instantaneous variation rate (higher value).

\begin{tabular}{lccccccc}
\hline$L(\mathrm{~km})$ & $\ell$ & $\left(\mathrm{km} \mathrm{yr}^{-1}\right)$ & $R_{m}$ & $\tau_{\max }(\mathrm{yr})$ & $\left(B_{z 0}^{\max }\right)^{\max }(\mu \mathrm{T})$ & $\mathrm{CMB}_{\left(\mu \mathrm{T} \mathrm{yr}^{-1}\right)}$ & $\operatorname{Surf}(\mu \mathrm{T} \mathrm{yr})$ \\
\hline 800 & 13 & 15 & 630 & 140 & $20 \times 10^{3}$ & $137-302$ & $0.016-0.035$ \\
2000 & 5 & 15 & 1590 & 410 & $31 \times 10^{3}$ & $75-182$ & $1.09-2.64$ \\
175 & 62 & 15 & 140 & 25 & $9 \times 10^{3}$ & $372-700$ & $0.6-1.1 \times 10^{-14} \approx 0$ \\
175 & 62 & 28 & 260 & 15 & $13 \times 10^{3}$ & $860-1726$ & $1.3-2.7 \times 10^{-14} \approx 0$ \\
\hline
\end{tabular}

secular variation in terms of Rossby waves in numerical dynamo simulations but stressed that this value could be much higher (up to approximately $10 \mathrm{mT}$ ). The results reported in this study for Configuration 3 suggest that the maximal proportion of $B_{z}$ expelled evolves as $\sim R_{m}^{1 / 2}$. Taking $3 \mathrm{mT}$ as the toroidal field strength in the vicinity of the CMB, the minimal and maximal lengths $L$ given above lead to an intensity of the expelled field of between $20 \mathrm{mT}$ $(L=800 \mathrm{~km})$ and $31 \mathrm{mT}\left(L=2 \times 10^{3} \mathrm{~km}\right)$. This corresponds to average intensity variation rates at the $\mathrm{CMB}$ of $75-137 \mu \mathrm{T} \mathrm{yr}^{-1}$. As for the maximum instantaneous rate $R^{\max }$, it reaches $302 \mu \mathrm{T} \mathrm{yr}^{-1}$ for $L=800 \mathrm{~km}$ and $182 \mu \mathrm{T} \mathrm{yr}^{-1}$ for $L=2000 \mathrm{~km}$, twice as much as the average rates. Assuming an attenuation of the field through the mantle on the order of $\sim\left(r_{c} / r_{a}\right)^{\ell+2}$, with $\ell$ the degree of the spherical harmonic decomposition, $r_{c}$ the radius of the core and $r_{a}$ the radius of the Earth, allows us to roughly estimate the intensity variation rates that would be observed at the Earth's surface. Such attenuation would lead to average intensity variation rates at Earth's surface of $\sim 0.016 \mu \mathrm{T} \mathrm{yr}^{-1}(L=800 \mathrm{~km})$ to $\sim 1.09 \mu \mathrm{T} \mathrm{yr}^{-1}(L=$ $2000 \mathrm{~km}, c f$. Table 2). Turning to the maximum instantaneous rate of change (eq. 10), using the same parameters than previously, the extrapolation leads to values ranging from 0.035 to $2.64 \mu \mathrm{T} \mathrm{yr}^{-1}$.

Having established geophysically plausible ranges for the rate of flux expulsion according to our simple model, it is now of interest to compare these with field intensity variations reported for geomagnetic spikes. It should first be mentioned that the intensity variation rates that have been associated with the latter, as well as their duration, are only approximate from an experimental point of view. On the basis of the available archeomagnetic data, it is not yet possible to know precisely whether the values that have been proposed characterize the spikes as a whole (with an estimate of the average variation rate and of the total duration of the rising or falling part of the intensities), or only part of the spikes. For this reason, in our discussion, we have considered both the average intensity variation rate and the maximum of the instantaneous rate of change over $\tau_{\max }$. Concerning the duration of geomagnetic spikes, it has been argued that their total duration may not exceed $\sim 50 \mathrm{yr}$, that is $\tau_{\max } \sim 25 \mathrm{yr}$ (Ben-Yosef et al. 2009; Shaar et al. 2011). Assuming the same characteristic velocity and magnetic diffusivity as used above, the observation of such a short lasting event would require a characteristic lengthscale of $\sim 175 \mathrm{~km}$ at the CMB and thus a local magnetic Reynolds number $R_{m} \sim 140$. These values would lead to a maximum amplitude of $9 \mathrm{mT}$ and an average variation rate at the $\mathrm{CMB}$ of $\sim 372 \mu \mathrm{T} \mathrm{yr}^{-1}$. The maximum instantaneous variation rate would reach $\sim 700 \mu \mathrm{T} \mathrm{yr}^{-1}$ in this case. However, and regardless of the variation rate we consider, such an event would not be detected at the Earth's surface, because of the geometric attenuation through the mantle, as it would correspond to degree $\ell \sim 62$ (the signal would be practically invisible at the Earth's surface).

From the scaling laws reported in eqs (15) and (16), a more general analysis can be performed to determine if geomagnetic field intensity peaks suggested by recent archeomagnetic data sets could be reasonably attributed to the signature at the Earth's surface of flux expulsion events at the CMB. As we aim to give optimistic estimates of the intensity rate of change produced by flux expulsion, we will therefore rely only on the results obtained from the scaling law of the maximum instantaneous rate $R^{\max }$, associated with the duration $\tau_{\max }$, corresponding to the time needed to reach the maximum of $B_{z 0}^{\max }$ (i.e. half the total duration of an intensity peak). Fig. 11 shows colour scale plots of the extrapolated maximum instantaneous variation rates at the Earth's surface as a function of the characteristic velocity and the characteristic wavelength of the process, for two different magnitudes of the toroidal field (3 and $10 \mathrm{mT}$, taken as plausible lower and upper values, see Hori et al. (2015)) and exploring two possible values of the magnetic diffusivity $\left(0.6\right.$ and $\left.2 \mathrm{~m}^{2} \mathrm{~s}^{-1}\right)$. The durations are represented by white dashed lines ( $\tau_{\max }$ being half of the total duration). The solid lines show values for variation rates, given in $\mu \mathrm{T} \mathrm{yr}^{-1}$. From this figure, it is observed that the dashed line corresponding to $\tau_{\max } \leq 50 \mathrm{yr}$ ( $\tau_{\text {tot }} \leq 100 \mathrm{yr}$ ), that is roughly two or three times the total duration initially suggested for geomagnetic spikes (Shaar et al. 2011), never cross the line corresponding to a variation rate of $4 \mu \mathrm{T} \mathrm{yr}^{-1}$. Shaar et al. (2016) and Ben-Yosef et al. (2017) suggest a less extreme geomagnetic spike during the 8th century $\mathrm{BC}$ that would be associated with variation rates of $\sim 0.75-1.5 \mu \mathrm{T} \mathrm{yr}^{-1}$. Considering the lowest intensity variation rate of $0.75 \mu \mathrm{T} \mathrm{yr}^{-1}$ and a total duration of $100 \mathrm{yr}$, flux expulsion could account for geomagnetic spikes only if the intensity of the toroidal magnetic field was increased to $10 \mathrm{mT}$ and if the lengthscale of the flow pattern was on the order of $1000 \mathrm{~km}$. Such values would however correspond to a flow speed greater than $50 \mathrm{~km} \mathrm{yr}^{-1}$ a rather extreme value according to inferences based on the present geomagnetic field (Hulot et al. 2002; Finlay \& Amit 2011; Holme 2015). For a total duration of $200 \mathrm{yr}\left(\tau_{\max }=100 \mathrm{yr}\right)$, a characteristic flow speed of $35 \mathrm{~km} \mathrm{yr}^{-1}$ would be needed. For the same duration, a variation rate as high as $4 \mu \mathrm{T} \mathrm{yr}^{-1}$ would require a characteristic flow speed of $50 \mathrm{~km} \mathrm{yr}^{-1}$. Adopting a higher value of the magnetic diffusivity $\left(2 \mathrm{~m}^{2} \mathrm{~s}^{-1}\right.$, Figs $11 \mathrm{c}$ and d) does not significantly change these results. To summarize, Fig. 11 clearly shows that according to our analyses magnetic flux expulsion can conceivably produce extreme variation rates only for large-scale events ( $>1000 \mathrm{~km}$ at the $\mathrm{CMB}$ ) and for a minimum total duration longer than a century.

Although less extreme, other periods characterized by strong intensity variation rates are proposed both in Western Europe and the Near-East. In the Near-East, Yutsis-Akimova et al. (2018a,b) report during the 6th millennium BC two intensity peaks of about one century or less (see also Kovacheva et al. 2014), associated with variation rates bounded between $\sim 0.1$ and $\sim 0.25 \mu \mathrm{T} \mathrm{yr}^{-1}$. Considering a total duration of $100 \mathrm{yr}$, a variation rate of $0.1 \mu \mathrm{T} \mathrm{yr}^{-1}$, and the commonly accepted value of $0.6 \mathrm{~m}^{2} \mathrm{~s}^{-1}$ for the magnetic diffusivity, such features would be reachable for a flow speed greater than $40 \mathrm{~km} \mathrm{yr}^{-1}$, more than twice its current rms value $\left(\sim 15 \mathrm{~km} \mathrm{yr}^{-1}\right)$. For the third millennium BC, Gallet et al. (2020) also report two 
(a)

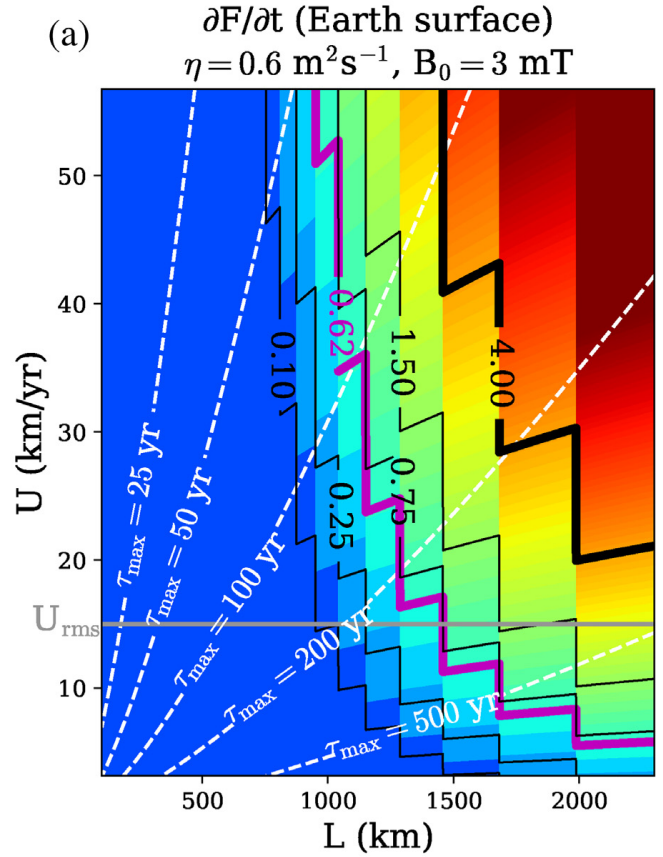

(c)

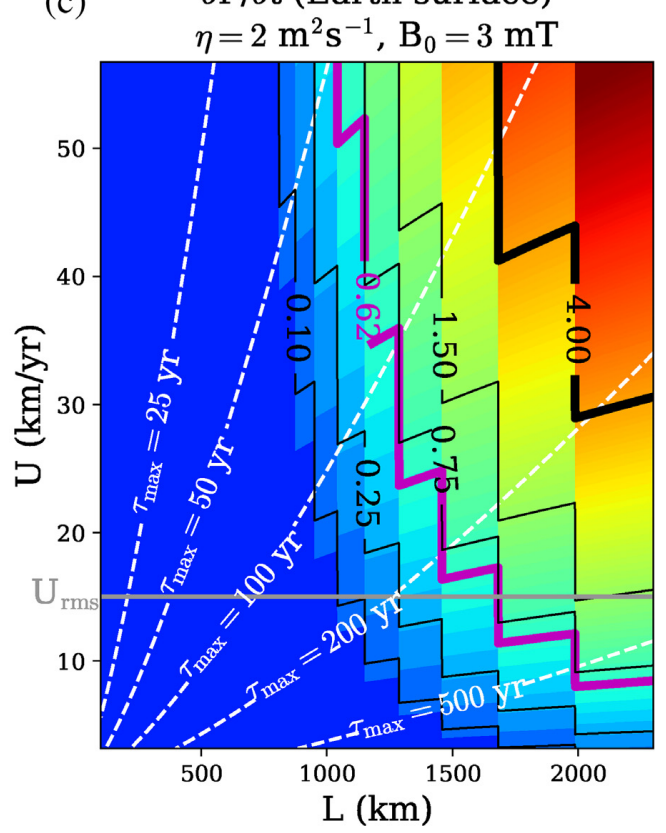

$\mu \mathrm{T} / \mathrm{yr}$
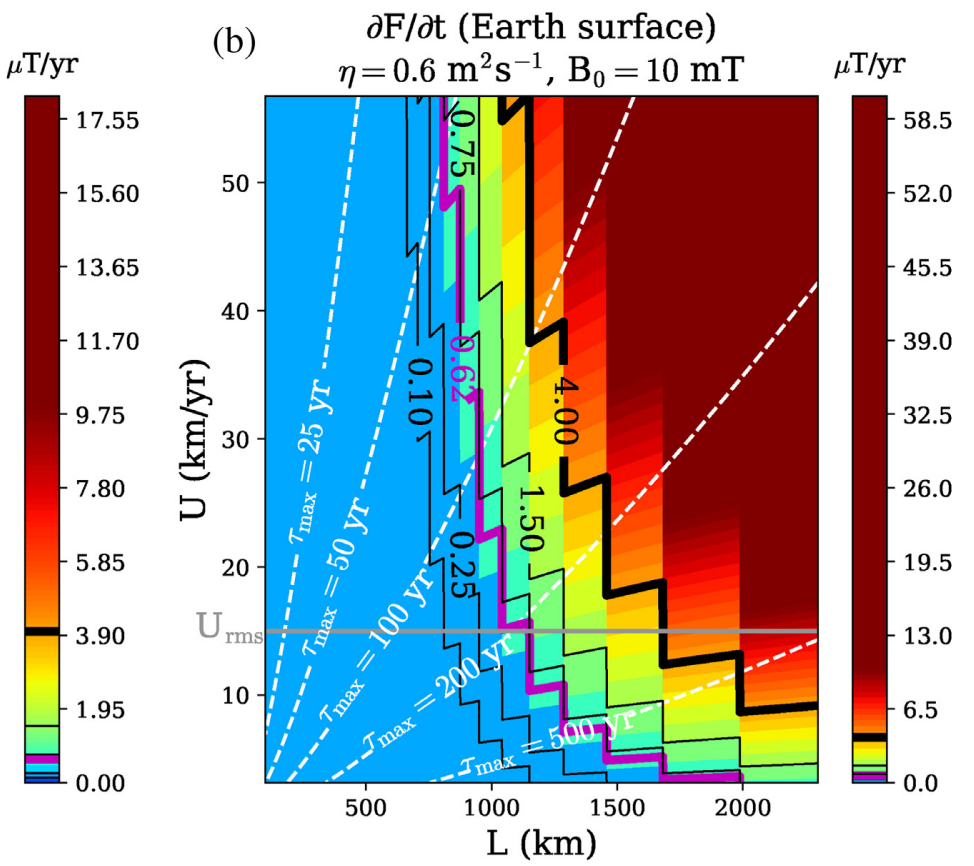

(d) $\quad \partial \mathrm{F} / \partial \mathrm{t}$ (Earth surface)

$\mu \mathrm{T} / \mathrm{yr}$

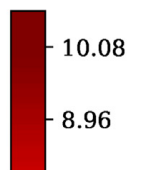

Figure 11. Instantaneous rate of change of the intensity of the field generated by flux expulsion as a function of the characteristic velocity of the fluid in the Earth's core and the characteristic scale of the process in the core, given for different initial intensity and magnetic diffusivity. $U_{\text {rms }}$ is the root mean square velocity at the top of the core. The solid lines represent some constant rates of change. The dashed lines give different values of $\tau_{\max }$ corresponding to half of the total duration of the expected peak of intensity induced by flux expulsion at the CMB.

intensity peaks in Mesopotamia associated with durations of $\sim 200$ $\mathrm{yr}$ and variation rates of $\sim 0.10-0.20 \mu \mathrm{T} \mathrm{yr}^{-1}$. These events would be compatible with flux expulsion over large spatial scale, with a reasonable flow speed of 20-30 $\mathrm{km} \mathrm{yr}^{-1}$. In Western Europe, Hervé et al. (2017) report a field intensity peak centred around $550 \mathrm{BC}$. This peak would have lasted about $400 \mathrm{yr}$ and is associated with variation rate of $\sim 0.25 \mu \mathrm{T} \mathrm{yr}^{-1}$ during its ascending branch ( 7 th century $\mathrm{BC})$, about twice the maximum value known in the modern field (Livermore et al. 2014). In this case, according to our experiments, such variations could be compatible with magnetic flux expulsion over large spatial scale ( $\sim 1000 \mathrm{~km}$ corresponding to degree 10$)$, associated with a flow speed of $15 \mathrm{~km} \mathrm{yr}^{-1}$, the rms flow speed at the CMB. Genevey et al. $(2013,2016)$ also show the existence in Western Europe of a series of geomagnetic field intensity peaks over the past $\sim 1500 \mathrm{yr}$. The total duration of these peaks is $\sim 200 \mathrm{yr}$ and they are associated with intensity variation rates from $\sim 0.05$ $\mu \mathrm{T} \mathrm{yr}^{-1}$, to a maximum of $\sim 0.15 \mu \mathrm{T} \mathrm{yr}^{-1}$, as for instance during the 10 th century AD. For such durations and variation rates, explaining these events by flux expulsion process appears also possible with a quite reasonable flow speed not exceeding 20-30 $\mathrm{km} \mathrm{yr}^{-1}$ and a 
lengthscale for the eddies that would approximately correspond to degree 13. This range of lengthscales for magnetic flux expulsion at the $\mathrm{CMB}$ would result in an extended large-scale signature at the Earth's surface, of about $1500-2000 \mathrm{~km}$ for $\ell \sim 10-13$.

Our analyses therefore lead to different interpretations of the geomagnetic intensity peaks reported in the Near-East and in Western Europe. In the Near-East, the peaks during the 6th millennium $\mathrm{BC}$ are obtained only for unrealistically high characteristic flow speeds (for the considered lengthscales and durations) while for the intensity peaks of the third millennium BC and those observed in Western Europe, they could reasonably be attributed to a flux expulsion process. It is worth recalling that the kinematic configuration considered in this study constitutes a particular limit (leading to high variation rates), for which the feedback of the Lorentz force on the velocity field is not considered, and the intensity variation rates estimated at the CMB are roughly extrapolated to the Earth's surface. Analysis of outputs from numerical dynamo simulations could help to determine whether flux expulsion events in a 3-D setting with self-consistent dynamics follow the scaling laws proposed here, or whether they are enhanced or attenuated by other processes.

\section{CONCLUSION}

The analysis of simple 2-D kinematic models of magnetic flux expulsion allows the determination of scaling laws governing the two physical processes involved in flux expulsion, namely the expulsion of magnetic flux from closed streamlines and subsequent expulsion through an insulating boundary, depending on the magnetic Reynolds number $R_{m}$.

Of the different cases studied here, Configuration 3 gives a better indication of the conditions prevailing in the outer core. For this configuration, we consider a rectangular domain containing two counter-rotating eddies with an insulating upper boundary. The maximum vertical component of the field that diffuses through the upper boundary $B_{z 0}^{\max }$ presents an intensity peak, characterized by a maximum amplitude reached over a time $\tau_{\max }$ which scales as $\sim R_{m}^{0.15}$. The maximum instantaneous rate of change scales as $\sim R_{m}^{0.45}$.

The extrapolation of the above scaling laws to the Earth (using reasonable estimates for the material properties and the flow magnitude at the $\mathrm{CMB}$ ) shows that extreme intensity variations rates proposed for geomagnetic spikes are difficult to obtain solely by magnetic flux expulsion. According to our experiments, extremely fast variations over a duration shorter than a century do not appear to be compatible with flux expulsion events. Durations and variation rates of more moderate intensity peaks detected in the Near-East and Western Europe during the Holocene are more compatible with our results.

An extension of this study to the dynamic case, considering the Lorentz force, would help to better characterize the expected signature of flux expulsion in the outer core. Dynamic variations rates are however expected to be lower than the kinematic ones we have investigated, since the feedback of the Lorentz force on the flow should lower the efficiency of expulsion (from the eddies and thereby through the insulating boundary). Recent studies of inverse geodynamo modelling covering the historical period during which direct measurements of the magnetic field are available (1840-2010) identify magnetic flux expulsion at low latitudes (Aubert 2014), arising from columnar vortices. To strengthen this conclusion, it would be interesting to look in details at outputs from numerical dynamos simulations presenting more realistic turbulent flows (Schaeffer et al. 2017) to seek magnetic flux expulsion events and study the underlying flow. It would also allow an assessment of whether the scaling laws determined in this study hold in the 3-D, fully dynamic case.

\section{ACKNOWLEDGEMENTS}

We thank two anonymous reviewers for their insightful comments that helped improve the paper. This work was supported by the Fondation Simone et Cino Del Duca of Institut de France (2017 Research Grant) and by the Programme National de Planétologie (PNP) of CNRS/INSU, co-funded by CNES. Numerical computations were performed on the S-CAPAD platform, IPGP, France. This is IPGP contribution 4131.

\section{REFERENCES}

Amit, H. \& Christensen, U., 2008. Accounting for magnetic diffusion in core flow inversions from geomagnetic secular variation, Geophys. J. Int., 175(3), 913-924.

Aubert, J., 2014. Earth's core internal dynamics 1840-2010 imaged by inverse geodynamo modelling, Geophys. J. Int., 197(3), 1321-1334.

Ben-Yosef, E., Tauxe, L., Levy, T., Shaar, R., Ron, H. \& Najjar, M., 2009. Geomagnetic intensity spike recorded in high resolution slag deposit in Southern Jordan, Earth planet. Sci. Lett., 287(3), 529-539.

Ben-Yosef, E., Millman, M., Shaar, R., Tauxe, L. \& Lipschits, O., 2017. Six centuries of geomagnetic intensity variations recorded by royal Judean stamped jar handles, Proc. Natl. Acad. Sci., 114(9), 2160-2165.

Bloxham, J., 1986. The expulsion of magnetic flux from the Earth's core, Geophys. J. Int., 87(2), 669-678.

Charbonneau, P., 2013. Solar and stellar dynamos, in Saas-Fee Advanced Course, Vol. 39, ed. Steiner, O., Springer.

Christensen, U. \& Wicht, J., 2015. Numerical dynamo simulations, in Treatise on Geophysics, 2nd edn, Vol. 8: Core Dynamics, pp. 245-277, Schubert, G., Elsevier B.V.

Christensen, U.R. \& Olson, P., 2003. Secular variation in numerical geodynamo models with lateral variations of boundary heat flow, Phys. Earth planet. Inter., 138(1), 39-54.

Chulliat, A., Hulot, G. \& Newitt, L., 2010. Magnetic flux expulsion from the core as a possible cause of the unusually large acceleration of the north magnetic pole during the 1990s, J. geophys. Res., 115(B7).

Davies, C. \& Constable, C., 2018. Searching for geomagnetic spikes in numerical dynamo simulations, Earth planet. Sci. Lett., 504, 72-83.

Drew, S., 1993. Magnetic field expulsion into a conducting mantle, Geophys. J. Int., 115(1), 303-312.

Finlay, C. \& Amit, H., 2011. On flow magnitude and field-flow alignment at Earth's core surface, Geophys. J. Int., 186(1), 175-192.

Finlay, C., Dumberry, M., Chulliat, A. \& Pais, M., 2010. Short timescale core dynamics: theory and observations, Space Sci. Rev., 155(1-4), 177-218.

Gallet, Y., Fortin, M., Fournier, A., Le Goff, M. \& Livermore, P., 2020. Analysis of geomagnetic field intensity variations in Mesopotamia during the third millennium BC with archeological implications, Earth planet. Sci. Lett., 537, 116183.

Galloway, D., Proctor, M. \& Weiss, N., 1978. Magnetic flux ropes and convection, J. Fluid Mech., 87(2), 243-261.

Genevey, A., Gallet, Y., Thébault, E., Jesset, S. \& Le Goff, M., 2013. Geomagnetic field intensity variations in Western Europe over the past 1100 years, Geochem. Geophys. Geosyst., 14(8), 2858-2872.

Genevey, A., Gallet, Y., Jesset, S., Thébault, E., Bouillon, J., Lefèvre, A. \& Le Goff, M., 2016. New archeointensity data from French Early Medieval pottery production (6th-10th century AD). tracing 1500 years of geomagnetic field intensity variations in Western Europe, Phys. Earth planet. Inter., 257, 205-219.

Gubbins, D., 1987. Mechanism for geomagnetic polarity reversals, Nature, 326(6109), 167-169. 
Gubbins, D., Jones, A. \& Finlay, C., 2006. Fall in Earth's magnetic field is erratic, Science, 312(5775), 900-902.

Hervé, G. et al., 2017. Fast geomagnetic field intensity variations between 1400 and 400 BCE: new archaeointensity data from Germany, Phys. Earth planet. Inter., 270, 143-156.

Holme, R., 2015. Large-scale flow in the core, in Treatise on Geophysics, 2nd edn, Vol. 8: Core Dynamics, pp. 161-181, ed. Schubert, G., Elsevier B.V.

Hori, K., Jones, C. \& Teed, R., 2015. Slow magnetic Rossby waves in the Earth's core, Geophys. Res. Lett., 42(16), 6622-6629.

Hulot, G., Eymin, C., Langlais, B., Mandea, M. \& Olsen, N., 2002. Smallscale structure of the geodynamo inferred from Oersted and Magsat satellite data, Nature, 416(6881), 620.

Jackson, A. \& Finlay, C., 2015. Geomagnetic secular variation and its applications to the core, in Treatise on Geophysics, 2nd edn, Vol. 5: Geomagnetism, ed. Schubert, G., pp. 137-184, Elsevier B.V.

Jones, C., 2015. Thermal and compositionnal convection in the outer core, in Treatise on Geophysics, 2nd edn, Vol. 8: Core Dynamics, ed. Schubert, G., pp. 115-159, Elsevier B.V.

Korte, M. \& Constable, C., 2018. Archeomagnetic intensity spikes: global or regional geomagnetic field features? Front. Earth Sci., $\mathbf{6}, 17$.

Kovacheva, M., Kostadinova-Avramova, M., Jordanova, N., Lanos, P. \& Boyadzhiev, Y., 2014. Extended and revised archaeomagnetic database and secular variation curves from Bulgaria for the last eight millennia, Phys. Earth planet. Inter., 236, 79-94.

Livermore, P., Fournier, A. \& Gallet, Y., 2014. Core-flow constraints on extreme archeomagnetic intensity changes, Earth planet. Sci. Lett., 387, 145-156.

Moffatt, H. \& Kamkar, H., 1983. On the time-scale associated with flux expulsion, in Stellar and Planetary Magnetism, ed. Soward, A.M., pp. 91-97, Gordon \& Breach Science Publishers Ltd.

Olsen, N., Lühr, H., Finlay, C., Sabaka, T., Michaelis, I., Rauberg, J. \& Tøffner-Clausen, L., 2014. The CHAOS-4 geomagnetic field model, Geophys. J. Int., 197(2), 815-827.

Pozzo, M., Davies, C., Gubbins, D. \& Alfe, D., 2012. Thermal and electrical conductivity of iron at Earth's core conditions, Nature, 485(7398), 355.

Rhines, P. \& Young, W., 1983. How rapidly is a passive scalar mixed within closed streamlines? J. Fluid Mech., 133, 133-145.

Roberts, P. \& Scott, S., 1965. On analysis of the secular variation, J. Geomag. Geoelectr., 17(2), 137-151.

Roberts, P.H., 1967. An Introduction to Magnetohydrodynamics, Longmans.

Schaeffer, N., Jault, D., Nataf, H.-C. \& Fournier, A., 2017. Turbulent geodynamo simulations: a leap towards Earth's core, Geophys. J. Int., 211(1), $1-29$.

Shaar, R., Ben-Yosef, E., Ron, H., Tauxe, L., Agnon, A. \& Kessel, R., 2011. Geomagnetic field intensity: How high can it get? How fast can it change? Constraints from Iron Age copper slag, Earth planet. Sci. Lett., 301(1), 297-306.

Shaar, R., Tauxe, L., Ron, H., Ebert, Y., Zuckerman, S., Finkelstein, I. \& Agnon, A., 2016. Large geomagnetic field anomalies revealed in Bronze to Iron Age archeomagnetic data from Tel Megiddo and Tel Hazor, Israel, Earth planet. Sci. Lett., 442, 173-185.

Thébault, E. et al., 2015. International geomagnetic reference field: the 12th generation, Earth, Planets Space, 67(1), 1-19.

Weiss, N.O., 1966. The expulsion of magnetic flux by eddies, Proc. R. Soc. Lond., A, 293(1434), 310-328.

Yutsis-Akimova, S., Gallet, Y. \& Amirov, S., 2018a. Rapid geomagnetic field intensity variations in the Near East during the 6th millennium BC: new archeointensity data from Halafian site Yarim Tepe II (Northern Iraq), Earth planet. Sci. Lett., 482, 201-212.

Yutsis-Akimova, S., Gallet, Y., Petrova, N., Nowak, S. \& Le Goff, M., 2018b. Geomagnetic field in the Near East at the beginning of the 6th millennium
BC: evidence for alternating weak and strong intensity variations, Phys. Earth planet. Inter., 282, 49-59.

\section{APPENDIX: NUMERICAL APPROXIMATION}

\section{A1 Numerical scheme and boundary conditions}

To numerically approximate eq. (3), we use a pseudospectral method involving a Fourier expansion along the $x$-direction together with a second-order accurate finite-difference scheme in the $z$-direction. The Fourier expansion depends on the lateral boundary conditions one intends to impose.

In configurations 1 and 2, magnetic field lines are anchored in the lateral boundaries. We decompose the vector potential $A$ according to

$A(x, z, t)=A_{0}(z)+A_{v}(x, z, t)$,

where $A_{0}(z)=A(x, z, t=0)$ is the initial vector potential (which is a function of $z$ only), and the time-dependent $A_{v}$ vanishes on the lateral boundaries, at $x=0$ and $x=L_{x}$. To satisfy exactly this vanishing requirement, $A_{v}$ is expanded in $x$ using sine functions. This amounts to rewriting eq. (A1) as

$A(x, z, t) \approx A_{0}(z)+\frac{1}{2 M} \sum_{k=0}^{M-1} \hat{A}_{v k}(z, t) \sin \left(\pi k \frac{x}{L_{x}}\right)$,

where $x \in] 0, L_{x}$ [ is discretely sampled at $M-1$ equally spaced internal points

$x_{k}=\frac{k L_{x}}{M}, \quad k=1, \ldots, M-1$,

and

$\hat{A}_{v k}(z, t) \equiv \sum_{m=1}^{M-1} A_{v}\left(x_{m}, z, t\right) \sin \left(\pi k \frac{m}{M}\right)$.

In configuration 3, magnetic field lines move freely along the lateral boundaries. The vector potential can then be approximated using a generic Fourier expansion, such that

$A(x, z, t) \approx \frac{1}{\sqrt{M}} \sum_{k=0}^{M-1} \hat{A}_{k}(z, t) \exp \left(-2 i \pi k \frac{x}{L_{x}}\right)$,

where $x \in\left[0, L_{x}\right.$ [is sampled at $M$ equally spaced points

$x_{k}=\frac{k L_{x}}{M}, \quad k=0,1, \ldots, M-1$,

and each Fourier mode $\hat{A}_{k}$ reads

$$
\begin{aligned}
\hat{A}_{k}(z, t) & \equiv \frac{1}{\sqrt{M}} \sum_{m=0}^{M-1} A\left(x_{m}, z, t\right) \exp \left(2 i \pi k \frac{m}{M}\right) \\
& \equiv \mathcal{F}_{k}[A(x, z, t)], \quad k=0,1, \ldots, M-1 .
\end{aligned}
$$

Regardless of the configuration, note that the $\hat{A}_{v k}$ and $\hat{A}_{k}$ undergo the same treatment. We will use the latter notation in the following to discuss in a common manner the treatment of configurations 1 , 2 and 3 .

If $\hat{\mathcal{J}}_{k}(z, t)$ denotes $\mathcal{F}_{k}[\mathcal{J}(x, z, t)]$, the Fourier expansion of eq. (3) yields 


$$
\begin{aligned}
& \frac{1}{\sqrt{M}} \sum_{k=0}^{M-1}\left\{\frac{\partial}{\partial t}-\frac{1}{R_{m}}\left[\frac{\partial^{2}}{\partial z^{2}}-k_{p}^{2}\right]\right\} \hat{A}_{k}(z, t) \exp \left(-2 i \pi k \frac{x}{L_{x}}\right) \\
& =\frac{1}{\sqrt{M}} \sum_{k=0}^{M-1} \hat{\mathcal{J}}_{k}(z, t) \exp \left(-2 i \pi k \frac{x}{L_{x}}\right) .
\end{aligned}
$$

with $k_{p}=2 \pi k / L_{x}$. The orthogonality of the Fourier basis then allows solving

$$
\left[\frac{\partial}{\partial t}-\frac{1}{R_{m}}\left(\frac{\partial^{2}}{\partial z^{2}}-k_{p}^{2}\right)\right] \hat{A}_{k}(z, t)=\hat{\mathcal{J}}_{k}(z, t),
$$

for each mode $k$.

Time $t \in[0, T]$ is sampled at discrete times $t_{n}=n \Delta t$ and the vertical direction $z \in\left[0, L_{z}\right]$ is sampled at a set of discrete $z_{j}=$ $j L_{z} /(N-1)=j \Delta z$. This prompts us to define $\hat{A}_{k}(j \Delta z, n \Delta t) \equiv \hat{A}_{k, j}^{n}$ and $\hat{\mathcal{J}}_{k}(j \Delta z, n \Delta t) \equiv \hat{\mathcal{J}}_{k, j}^{n}$. The centred finite difference scheme in $z$ yields

$$
\begin{aligned}
& \frac{\hat{A}_{k, j}^{n+1}-\hat{A}_{k, j}^{n}}{\Delta t}-\frac{1}{R_{m}}\left[\frac{\hat{A}_{k, j+1}^{n+1}-2 \hat{A}_{k, j}^{n+1}+\hat{A}_{k, j-1}^{n+1}}{2 \Delta z^{2}}\right. \\
& +\frac{\left.\hat{A}_{k, j+1}^{n}-2 \hat{A}_{k, j}^{n}+\hat{A}_{k, j-1}^{n}-\frac{k_{p}^{2}}{2}\left(\hat{A}_{k, j}^{n+1}+\hat{A}_{k, j}^{n}\right)\right]=\hat{\mathcal{J}}_{k, j}^{n}(\mathrm{~A})}{2 \Delta z^{2}}
\end{aligned}
$$

for the interior points $z_{j}, j=1, \ldots, N-2$. This equation is modified near the boundaries in order to accommodate boundary conditions.

The lower boundary $(j=N-1)$ is supposed to be perfectly conducting for all three configurations. As the field in a perfectly conducting medium is static, the vector potential for all non-zero modes is forced to zero at the lower boundary, and the $k=0$ mode remains equal to its initial value

$$
\begin{aligned}
& \hat{A}_{k, N-1}^{n}=0 \forall k \neq 0, \forall n, \\
& \hat{A}_{0, N-1}^{n}=\hat{A}_{0, N-1}^{0} \forall n .
\end{aligned}
$$

In configuration 1 , the top is also perfectly conducting. The same condition is then applied

$\hat{A}_{k, 0}^{n}=0 \forall k \neq 0, \forall n$,

$\hat{A}_{0,0}^{n}=\hat{A}_{0,0}^{0} \forall n$.

If $\mathbf{A}_{k}^{n}$ denotes the column vector of unknowns $\left[\hat{A}_{k, 0}^{n}, \hat{A}_{k, 1}^{n}, \ldots, \hat{A}_{k, N-2}^{n}, \hat{A}_{k, N-1}^{n}\right]^{T}$ (in which $T$ implies transposition), the linear system to solve at each time step for configuration 1 reads

$\mathbf{M}_{k}^{(1)} \mathbf{A}_{k}^{n+1}=\mathbf{N}_{k}^{(1)} \mathbf{A}_{k}^{n}+\mathbf{J}_{k}^{n}=\mathbf{F}_{k}^{n}$,

where
$\mathbf{M}_{k}^{(1)}=\left[\begin{array}{ccccccc}1 & 0 & 0 & \cdots & \cdots & \cdots & 0 \\ 0 & \gamma_{k} & \rho & \ddots & & & \vdots \\ 0 & \rho & \ddots & \ddots & \ddots & & \vdots \\ \vdots & \ddots & \ddots & \gamma_{k} & \ddots & \ddots & \vdots \\ \vdots & & \ddots & \ddots & \ddots & \rho & 0 \\ \vdots & & & \ddots & \rho & \gamma_{k} & 0 \\ 0 & \cdots & \cdots & \cdots & 0 & 0 & 1\end{array}\right], \forall k$,

with $\gamma_{k}=\frac{1}{\Delta t}+\frac{1}{R_{m}}\left(\frac{1}{\Delta z^{2}}+\frac{k_{p}^{2}}{2}\right)$ and $\rho=-\frac{1}{R_{m}} \frac{1}{2 \Delta z^{2}}$;

$\mathbf{N}_{k}^{(1)}=\left[\begin{array}{ccccccc}b_{k} & 0 & 0 & \cdots & \cdots & \cdots & 0 \\ 0 & \chi_{k} & -\rho & \ddots & & & \vdots \\ 0 & -\rho & \ddots & \ddots & \ddots & & \vdots \\ \vdots & \ddots & \ddots & \chi_{k} & \ddots & \ddots & \vdots \\ \vdots & & \ddots & \ddots & \ddots & -\rho & 0 \\ \vdots & & & \ddots & -\rho & \chi_{k} & 0 \\ 0 & \cdots & \cdots & \cdots & 0 & 0 & b_{k}\end{array}\right], \forall k$,

with $\chi_{k}=\frac{1}{\Delta t}-\frac{1}{R_{m}}\left(\frac{1}{\Delta z^{2}}+\frac{k_{p}^{2}}{2}\right) ; b_{0}=1$ and $b_{k}=0$ for $k \neq 0$, leading to

$$
\begin{aligned}
\mathbf{F}_{0}^{n}= & {\left[\hat{A}_{0,0}^{0}, \chi_{0} \hat{A}_{0,1}^{n}-\rho \hat{A}_{0,2}^{n}+\hat{\mathcal{J}}_{0,1}^{n}+\frac{\hat{A}_{0,0}^{0}}{R_{m} \Delta z^{2}}, \ldots,\right.} \\
& -\rho \hat{A}_{0, j-1}^{n}+\chi_{0} \hat{A}_{0, j}^{n}-\rho \hat{A}_{0, j+1}^{n}+\hat{\mathcal{J}}_{0, j}^{n}, \ldots, \\
& \left.-\rho \hat{A}_{0, N-3}^{n}+\chi_{0} \hat{A}_{0, N-2}^{n}+\hat{\mathcal{J}}_{0, N-2}^{n}+\frac{\hat{A}_{0, N-1}^{0}}{R_{m} \Delta z^{2}}, \hat{A}_{0, N-1}^{0}\right]^{T}
\end{aligned}
$$

for the $k=0$ mode and

$$
\begin{aligned}
\mathbf{F}_{k}^{n}= & {\left[0, \chi_{k} \hat{A}_{k, 1}^{n}-\rho \hat{A}_{k, 2}^{n}+\hat{\mathcal{J}}_{k, 1}^{n}, \ldots,\right.} \\
& -\rho \hat{A}_{k, j-1}^{n}+\chi_{k} \hat{A}_{k, j}^{n}-\rho \hat{A}_{k, j+1}^{n}+\hat{\mathcal{J}}_{k, j}^{n}, \ldots, \\
& \left.-\rho \hat{A}_{k, N-3}^{n}+\chi_{k} \hat{A}_{k, N-2}^{n}+\hat{\mathcal{J}}_{k, N-2}^{n}, 0\right]^{T}
\end{aligned}
$$

for each $k \neq 0$ mode.

In configurations 2 and 3, the top boundary is insulating. By using the fact that the magnetic field in the $z<0$ region is a potential field, Bloxham (1986) showed that the corresponding boundary condition reads

$k_{p} \hat{A}_{k}(z, t)=\frac{\partial \hat{A}_{k}(z, t)}{\partial z}, \quad \forall k \neq 0$.

Using a centred finite difference scheme, this becomes

$\hat{A}_{k,-1}^{n}=\hat{A}_{k, 1}^{n}-2 k_{p} \Delta z \hat{A}_{k, 0}^{n}$.

using a ghost node located at $z_{-1}=-\Delta z$. For $k=0$, we impose 
$\hat{A}_{0,0}^{n}=0$.

Adopting the same notations than for Configuration 1 (eq. A14), the components of the linear system to solve for Configuration $2 / 3$ become

$\mathbf{M}_{k}^{(2 / 3)}=\left[\begin{array}{ccccccc}\xi_{k} & 2 \rho & 0 & \cdots & \cdots & \cdots & 0 \\ \rho & \gamma_{k} & \rho & \ddots & & & \vdots \\ 0 & \rho & \ddots & \ddots & \ddots & & \vdots \\ \vdots & \ddots & \ddots & \gamma_{k} & \ddots & \ddots & \vdots \\ \vdots & & \ddots & \ddots & \ddots & \rho & 0 \\ \vdots & & & \ddots & \rho & \gamma_{k} & 0 \\ 0 & \cdots & \ldots & \cdots & 0 & 0 & 1\end{array}\right], k \neq 0$

with $\xi_{k}=\frac{1}{\Delta t}+\frac{1}{R_{m}}\left(\frac{1+k_{p} \Delta z}{\Delta z^{2}}+\frac{k_{p}^{2}}{2}\right)$;

$\mathbf{M}_{0}^{(2 / 3)}=\left[\begin{array}{ccccccc}1 & 0 & 0 & \cdots & \cdots & \cdots & 0 \\ 0 & \gamma_{0} & \rho & \ddots & & & \vdots \\ 0 & \rho & \ddots & \ddots & \ddots & & \vdots \\ \vdots & \ddots & \ddots & \gamma_{0} & \ddots & \ddots & \vdots \\ \vdots & & \ddots & \ddots & \ddots & \rho & 0 \\ \vdots & & & \ddots & \rho & \gamma_{0} & 0 \\ 0 & \cdots & \cdots & \cdots & 0 & 0 & 1\end{array}\right]$

$\mathbf{N}_{k}^{(2 / 3)}=\left[\begin{array}{ccccccc}\zeta_{k} & -2 \rho & 0 & \cdots & \cdots & \cdots & 0 \\ -\rho & \chi_{k} & -\rho & \ddots & & & \vdots \\ 0 & -\rho & \ddots & \ddots & \ddots & & \vdots \\ \vdots & \ddots & \ddots & \chi_{k} & \ddots & \ddots & \vdots \\ \vdots & & \ddots & \ddots & \ddots & -\rho & 0 \\ \vdots & & & \ddots & -\rho & \chi_{k} & 0 \\ 0 & \ldots & \ldots & \cdots & 0 & 0 & 1\end{array}\right], k \neq 0$

with $\zeta_{k}=\frac{1}{\Delta t}-\frac{1}{R_{m}}\left(\frac{1+k_{p} \Delta z}{\Delta z^{2}}+\frac{k_{p}^{2}}{2}\right)$

$\mathbf{N}_{0}^{(2 / 3)}=\left[\begin{array}{ccccccc}0 & 0 & 0 & \cdots & \cdots & \cdots & 0 \\ 0 & \chi_{0} & -\rho & \ddots & & & \vdots \\ 0 & -\rho & \ddots & \ddots & \ddots & & \vdots \\ \vdots & \ddots & \ddots & \chi_{0} & \ddots & \ddots & \vdots \\ \vdots & & \ddots & \ddots & \ddots & -\rho & 0 \\ \vdots & & & \ddots & -\rho & \chi_{0} & 0 \\ 0 & \cdots & \cdots & \cdots & 0 & 0 & 1\end{array}\right]$

leading to

$$
\begin{aligned}
\mathbf{F}_{k}^{n}= & {\left[\zeta_{k} \hat{A}_{k, 0}^{n}-2 \rho \hat{A}_{k, 1}^{n},-\rho \hat{A}_{k, 0}^{n}+\chi_{k} \hat{A}_{k, 1}^{n}-\rho \hat{A}_{k, 2}^{n}+\hat{\mathcal{J}}_{k, 1}^{n}, \ldots\right.} \\
& -\rho \hat{A}_{k, j-1}^{n}+\chi_{k} \hat{A}_{k, j}^{n}-\rho \hat{A}_{k, j+1}^{n}+\hat{\mathcal{J}}_{k, j}^{n}, \ldots \\
& \left.-\rho \hat{A}_{k, N+2 N-3}^{n}+\chi_{k} \hat{A}_{k, N-2}^{n}+\hat{\mathcal{J}}_{k, N-2}^{n}, 0\right]^{T}
\end{aligned}
$$

for each $k \neq 0$ mode and

$$
\begin{aligned}
\mathbf{F}_{0}^{n}= & {\left[0, \chi_{0} \hat{A}_{0,1}^{n}-\rho \hat{A}_{0,2}^{n}+\hat{\mathcal{J}}_{0,1}^{n}, \ldots,\right.} \\
& -\rho \hat{A}_{0, j-1}^{n}+\chi_{0} \hat{A}_{0, j}^{n}-\rho \hat{A}_{0, j+1}^{n}+\hat{\mathcal{J}}_{0, j}^{n}, \ldots, \\
& \left.-\rho \hat{A}_{0, N-3}^{n}+\chi_{0} \hat{A}_{0, N-2}^{n}+\hat{\mathcal{J}}_{0, N-2}^{n}+\frac{\hat{A}_{0, N-1}^{0}}{R_{m} \Delta z^{2}}, \hat{A}_{0, N-1}^{0}\right]^{T}
\end{aligned}
$$

for the $k=0$ mode, respectively.

This pseudospectral method has been implemented using python and its linear algebra and Fourier transform libraries.

\section{A2 Validation tests}

\section{A2.1 Diffusive limit: theoretical solution inside the domain}

Drew (1993) tested his numerical results for the diffusion equation

$$
\frac{\partial \mathbf{B}}{\partial t}=\nabla^{2} \mathbf{B}
$$

in the case of a perfectly conducting boundary at the bottom and an insulating boundary at the top. For a purely horizontal initial field $\mathbf{B}=B(z, t) \hat{\boldsymbol{x}}$, this equation becomes scalar

$\frac{\partial B(z, t)}{\partial t}=\frac{\partial^{2} B(z, t)}{\partial z^{2}}$,

subject to the boundary conditions,

$\frac{\partial B(z, t)}{\partial z}=0$ at $z=1, \forall t$,

$B(z, t)=0$ at $z=0, \forall t$.

Assuming an exponential decay $e^{-p t}$ of the field gives the following diffusive modes

$B_{p}(z, t)=B_{0} \cos [\sqrt{p}(1-z)] e^{-p t}, \forall p$

with $B_{0}$ a constant and $\sqrt{p}=\frac{(2 q+1) \pi}{2}, q=0,1,2, \ldots$ the decay rate. The fundamental $(q=0)$ mode is

$B_{p 0}(z, t)=B_{0} \cos \left[\frac{\pi}{2}(1-z)\right] e^{-\frac{\pi^{2}}{4} t}$.

The problem is solved using the same set of parameters than those used by Drew (1993), with $L_{z}=L=1$ and $L_{x}=2 L=2$. Using our method, the magnetic field is expressed in terms of the vector potential in the Fourier domain. Consequently, the condition

$\hat{A}_{p}(z=1, t)=0 \quad \forall t, \forall p$

is imposed at the bottom. At the top, an insulating boundary is specified for all modes as follows

$\frac{\partial \hat{A}_{p}(z=0, t)}{\partial z}=0 \quad \forall t, \forall p$.

The initial field is set to $B_{p}(z, t=0)=\cos [\sqrt{p}(1-z)]$ and in terms of vector potential

$A_{p}(z, t=0)=\frac{1}{\sqrt{p}} \sin [\sqrt{p}(1-z)] \quad \forall p$.

Calculations are carried out using a time step size $\Delta t=1.10^{-4}$ and $N=200$ points in the $z$ direction. Fig. A1 shows the evolution of the vector potential at the centre of the domain $\left(x_{c}=1, z_{c}=0.5\right)$, for the analytical (straight lines) and the numerical (symbols) solutions as a function of time (expressed in advection time units). The slopes for the different modes give the corresponding decay coefficient $p$. 


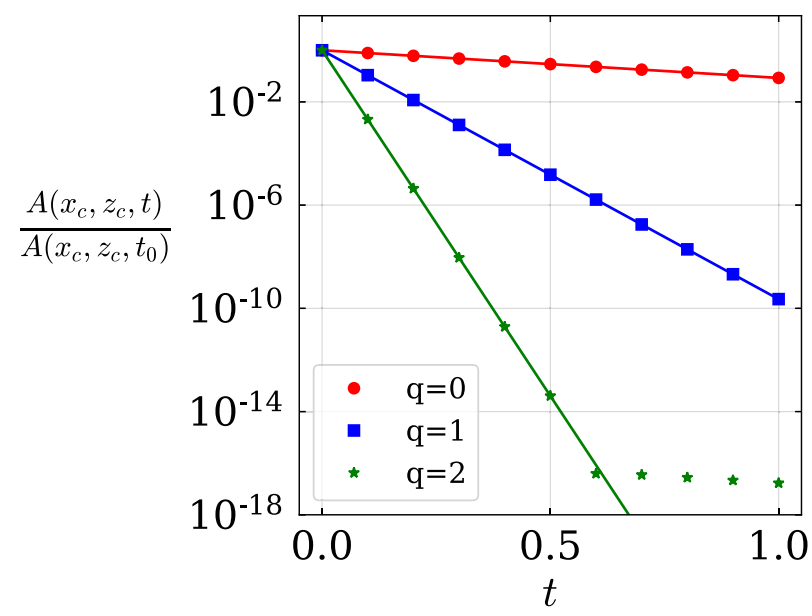

Figure A1. Decay of the vector potential in the centre of the layer as a function of time (expressed in advection time units) for the first three diffusive modes computed for $N=200$ and $\Delta t=1.10^{-4}$.

Table A1. Theoretical and numerical values of the decay coefficient of the vector potential for different modes $q$ (for $q=2$, as the numerical solution reaches the numerical accuracy after $t=0.6$, see Fig. A1, the slope is computed for the previous time steps, from $t=0$ to $t=0.6$ ).

\begin{tabular}{lcc}
\hline & $\sqrt{p}$ theo & $\sqrt{p}$ num \\
\hline$q=0$ & 1.57079633 & $1.57078058 \pm 2.26 \times 10^{-15}$ \\
$q=1$ & 4.71238898 & $4.71204979 \pm 9.69 \times 10^{-8}$ \\
$q=2$ & 7.85398163 & $7.93229119 \pm 1.76 \times 10^{-1}$ \\
\hline
\end{tabular}

For the fundamental mode $q=0$, the numerical solution gives a decay coefficient very close to the theoretical expectation $(\sqrt{p}=$ $1.5708)$. The accuracy of the numerical computation is also checked for modes corresponding to $q=1$ and $q=2$ (cf. Table A1). Again, the numerical solution is very close to the analytical solution, although not as close as for the $q=0$ mode.

We define the average cumulative error $e$

$e=\frac{1}{T} \int_{0}^{T}\left\{\frac{1}{\mathcal{D}} \iint_{\mathcal{D}}\left[A_{\text {ref }}(z, t)-A_{\text {num }}(z, t)\right]^{2} \mathrm{~d} \mathcal{D}\right\}^{\frac{1}{2}} \mathrm{~d} t$,

with $A_{\text {ref }}(z, t)$ and $A_{\text {num }}(z, t)$ the analytical and numerical solutions, respectively; $\mathcal{D}$ is the domain and $T$ the integration time. This error is computed for different sets of $\Delta t$ and $\Delta z$. Results are shown in Fig. A2.

As expected, with decreasing $\Delta t$ and $\Delta z$, the error tends to zero, in an algebraic fashion. In either case, the slope is close to 2 , as expected for the Crank-Nicholson scheme in time and the centred finite difference scheme we chose in space.

\section{A2.2 Diffusive limit: analytical solution in the case of an insulating upper boundary condition}

In order to assess the numerical treatment of the insulating boundary condition (see eqs A20 and A21), an analytical solution for the diffusion equation is determined for a domain $\mathcal{D}$ of aspect ratio 2 : 1 , with a insulating top boundary and a perfectly conducting bottom boundary. The Fourier expansion of the diffusion equation for the vector potential is

$\left[\frac{\partial}{\partial t}-\left(\frac{\partial^{2}}{\partial z^{2}}-k_{p}^{2}\right)\right] \hat{A}_{k}(z, t)=0$, with $R_{m}=1$ and $k_{p}=\pi k / L_{x} \forall k$. This equation can be solved for each mode $k$.

As this is a simple diffusion problem, we apply a root finding procedure. To satisfy the boundary conditions, we have to solve

$\tan (\mu L)=\frac{-\mu}{k_{p}}$,

with $\mu=\sqrt{-\left(\lambda+k_{p}^{2}\right)}$ and $\lambda+k_{p}^{2}<0$, with $\lambda<0$ a decay time coefficient. Let $\lambda_{m}$ denote the $m$-th eigenvalue for $m=1,2,3, \ldots$

The solution of eq. (A38) is given by

$\hat{A}_{k}(z, t)=\sum_{m} E_{m} e^{\lambda_{m} t}\left[\frac{\mu_{m}}{k_{p}} \cos \left(z \mu_{m}\right)+\sin \left(z \mu_{m}\right)\right]$,

with $\mu_{m}=\sqrt{-\left(\lambda_{m}+k_{p}^{2}\right)}$. At $t=0$, eq. (A40) becomes

$\hat{A}_{k}(z, t=0)=\sum_{m} E_{m}\left[\frac{\mu_{m}}{k_{p}} \cos \left(z \mu_{m}\right)+\sin \left(z \mu_{m}\right)\right]=\sum_{m} E_{m} G_{m}$.

Multiplying with $G_{m^{\prime}}$ and integrating over $z$ gives:

$\int_{0}^{1} \hat{A}_{k}(z, t=0) G_{m^{\prime}} \mathrm{d} z=\int_{0}^{1} \sum_{m} E_{m} G_{m} G_{m^{\prime}} \mathrm{d} z$.

The orthogonality of the eigenfunctions leads to $G_{m} G_{m^{\prime}}=0$ if $m$ $\neq m^{\prime}$ and therefore

$\int_{0}^{1} \hat{A}_{k}(z, t=0) G_{m} \mathrm{~d} z=E_{m} \int_{0}^{1} G_{m}^{2} \mathrm{~d} z$,

which leads to

$E_{m}=\frac{\int_{0}^{1} \hat{A}_{k}(z, t=0) G_{m} \mathrm{~d} z}{\int_{0}^{1} G_{m}^{2} \mathrm{~d} z}$.

Let us define the initial field as

$\hat{A}_{k}(z, t=0)=\frac{\mu_{m}}{k_{p}} \cos \left(z \mu_{m}\right)+\sin \left(z \mu_{m}\right)$,

and focus on the $m=1$ case. Fig. A3 shows the evolution of the numerical and theoretical solutions at the centre of the domain $\left(x_{c}\right.$ $\left.=1, z_{c}=0.5\right)$, precisely for $m=1$, taking a time step size $\Delta t=$ $10^{-3}$ and 200 points in the $z$ direction. Both exhibit a decrease of the amplitude, following the decay rate $-\lambda_{1}$. The numerical value of $\lambda_{1}, \lambda_{\text {num }}=-15.992412$ is close to the theoretical expectation $\lambda_{\text {theo }}=-15.991856$.

The components of the magnetic field are

$B_{x}(x, z, t)=-\frac{\partial A(x, z, t)}{\partial z}$,

$B_{z}(x, z, t)=\frac{\partial A(x, z, t)}{\partial x}$.

In the Fourier domain the latter expands as

$\hat{B}_{z k}(z, t)=-i k_{p} \hat{A}_{k}(z, t)$.

Using eq. (A40), this yields

$\hat{B}_{z k}(z, t)=-i k_{p} \sum_{m} E_{m} e^{\lambda_{m} t}\left[\frac{\mu_{m}}{k_{p}} \cos \left(z \mu_{m}\right)+\sin \left(z \mu_{m}\right)\right]$.

To assess the imposition of the insulating top boundary condition, an average cumulative error is computed using a diagnostic similar to eq. (A37), restricted to the upper boundary for the $z$-component 

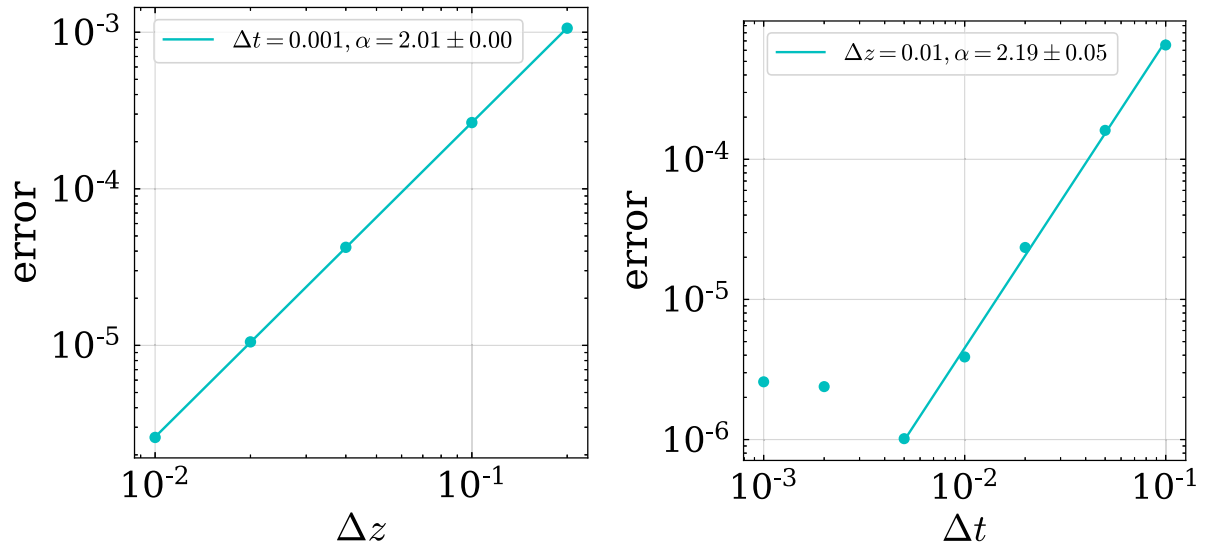

Figure A2. Convergence analysis for the fundamental mode $q=0$. Left-hand panel: error as a function of the grid size $\Delta z$. Right-hand panel: error as a function of the time step $\Delta t$. The slope of the straight line is the order of the numerical scheme in the $z$-direcion (left-hand panel) and in time (right-hand panel).

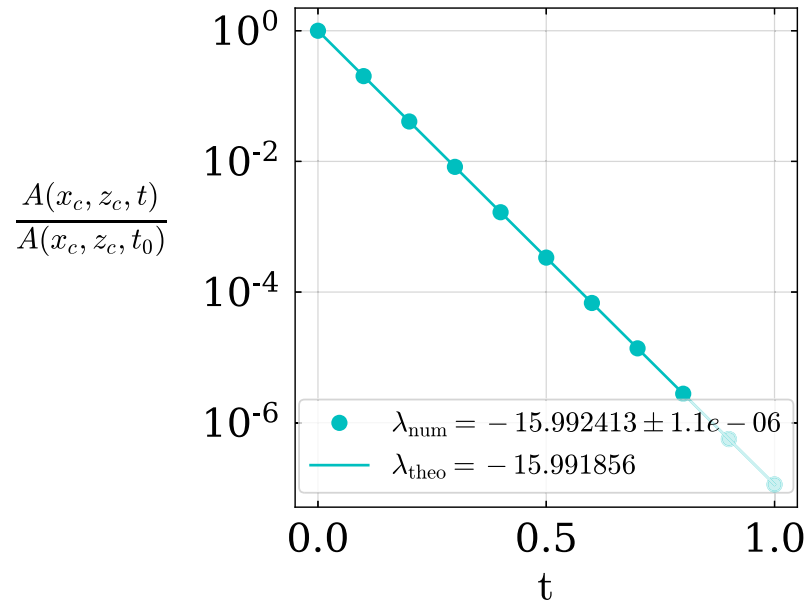

Figure A3. Decay time for the 0 mode computed for a model with $N=200$ and $\Delta t=1 \cdot 10^{-3} \cdot A\left(x_{c}, z_{c}, t\right)$ is the value of the vector potential at the centre of the layer, normalised by its initial value $A\left(x_{c}, z_{c}, t=0\right)$.

of the field

$$
\begin{gathered}
e=\frac{1}{T} \int_{0}^{T}\left\{\frac { 1 } { L _ { x } } \int _ { x = 0 } ^ { x = L _ { x } } \left[B_{z, \text { ref }}(x, z=0, t)\right.\right. \\
\left.\left.-B_{z, \text { num }}(x, z=0, t)\right]^{2} \mathrm{~d} x\right\}^{\frac{1}{2}} \mathrm{~d} t,
\end{gathered}
$$

As previously, Fig. A4 shows that the numerical scheme is as expected of order 2 in both space and time.

\section{A2.3 Advective limit}

In order to test the numerical approximation of the advection term in the induction equation (eq. 2) and to check that the time evolution is well-controlled in our modelling, we study the evolution of the vector potential in a domain $\mathcal{D}$ of aspect ratio $2: 1$, periodic along the $x$-direction, with perfectly conducting upper and lower boundaries. A high magnetic Reynolds number $R_{m}=10^{5}$ is chosen to mimic a diffusionless setting. The initial magnetic field is a Gaussian bell of the form

$A(x, z, t=0)=\exp \left[-\frac{\left(x-x_{0}\right)^{2}+\left(z-z_{0}\right)^{2}}{2 \sigma^{2}}\right]$,

and the imposed velocity field is

$u_{x}=-\frac{\partial \Psi}{\partial z}=-U_{0} \hat{\boldsymbol{x}}$

$u_{z}=\frac{\partial \Psi}{\partial x}=0$

which amounts to a translation in the $x$ direction.

The initial state is represented in Fig. A5. The magnetic field takes the form of a bell whose size is controlled by the amplitude of $\sigma$ in the Gaussian function. In order to meet the boundary conditions, we require $\sigma \ll L_{z}$. Here we choose to impose $\sigma=0.1 L_{z}$, with $x_{0}$ $=L$ and $z_{0}=0.5 L$. Taking the periodicity of the problem along the $x$-direction into account, with the chosen velocity field, the bell is expected to get back to its initial position after one advection time $\tau_{\text {adv }}$. Fig. A5 depicts an evolution in line with what is expected in this configuration. To assess the order of the scheme, we define the error as

$e=\max \left(\left|A-A_{\text {ref }}\right|\right)$

with $A=A(x, z, t=1)$ and $A_{\text {ref }}=A(x, z, t=0)$. The error is computed for different $\Delta z$ and $\Delta t$. Results are given in Fig. A6. As expected for the advection term, the scheme is of order 1 in time. For very small $\Delta t$ the error increases as $\Delta t$ decreases. The imposed $R_{m}$ is finite (though very small) which means that, at a certain point, diffusion eventually occurs, preventing the error to decrease with smaller $\Delta t$ as expected. 

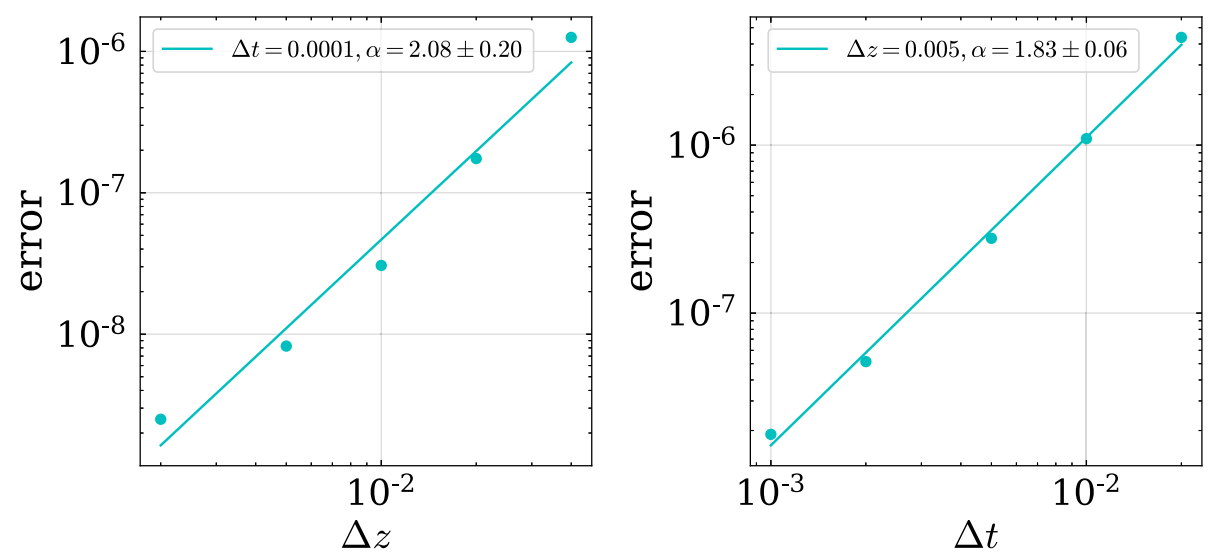

Figure A4. Convergence analysis for $B_{z}$ in $z=0$. Left-hand panel: error as a function of the grid size $\Delta z$, right-hand panel: error as a function of the time step $\Delta t$. The $\alpha$ value gives the computed slope for each case. 

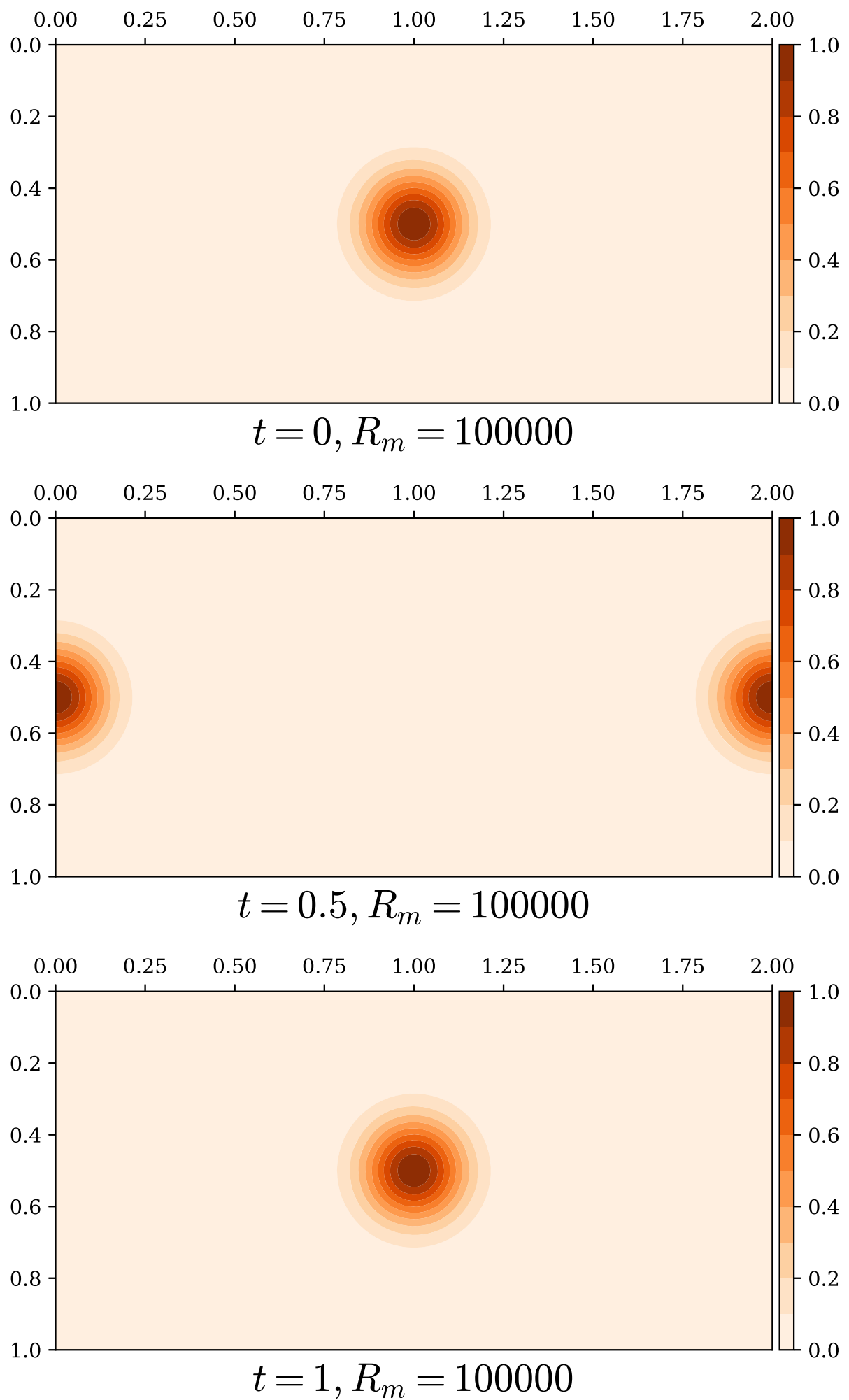

Figure A5. Evolution of a Gaussian-shaped vector potential. The upper, middle and lower panels shows vector potential at the initial time, after one half of an advection time and one advection time, respectively. The colour scale represents the intensity of the magnetic field. 


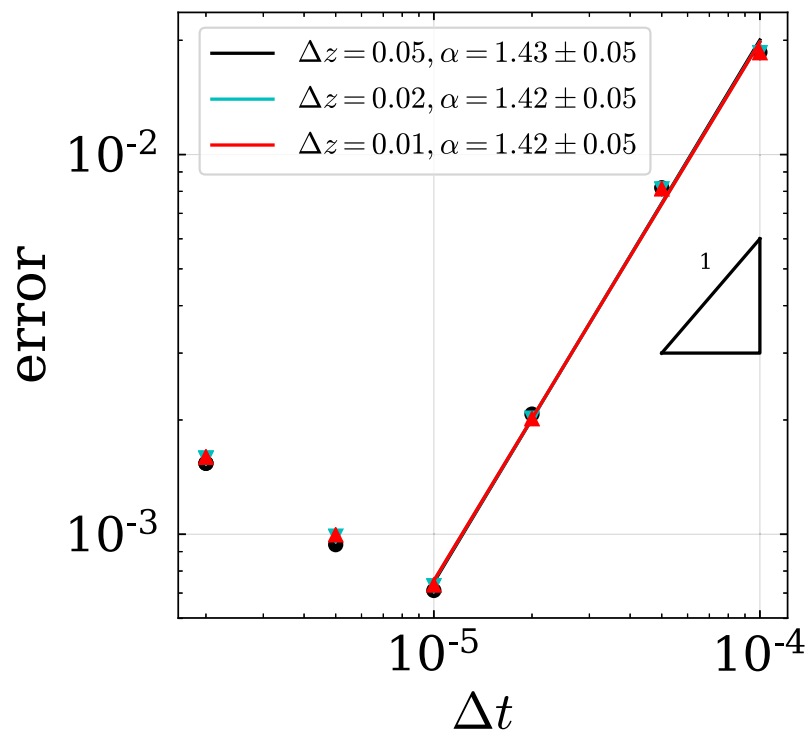

Figure A6. Convergence analysis for the advective component of the induction equation. The error is represented as a function of the time step $\Delta t$. Each straight line of slope $\alpha$ is obtained for each $\Delta z$ by least-squares fitting. 